\title{
The matching polynomials and spectral radii of uniform supertrees*
}

\author{
$\mathrm{Li} \mathrm{Su}^{1,2} \quad$ Liying Kang ${ }^{1 \dagger}$ \\ ${ }^{1}$ Department of Mathematics \\ Shanghai University \\ Shanghai 200444, PR China \\ suli@jxnu.edu.cn, lykang@i.shu.edu.cn \\ Honghai $\mathrm{Li}^{2 \ddagger}$ \\ ${ }^{2}$ College of Mathematics and Information Science \\ Jiangxi Normal University \\ Nanchang, Jiangxi 330022, PR China \\ lhh@mail.ustc.edu.cn \\ Erfang Shan ${ }^{\S}$ \\ School of Management \\ Shanghai University \\ Shanghai 200444, PR China \\ ef shan@shu.edu.cn
}

Submitted: Apr 30, 2018; Accepted: Sep 23, 2018; Published: Oct 19, 2018

(C) The authors. Released under the CC BY-ND license (International 4.0).

\begin{abstract}
We study matching polynomials of uniform hypergraph and spectral radii of uniform supertrees. By comparing the matching polynomials of supertrees, we extend $\mathrm{Li}$ and Feng's results on grafting operations on graphs to supertrees. Using the methods of grafting operations on supertrees and comparing matching polynomials of supertrees, we determine the first $\left\lfloor\frac{d}{2}\right\rfloor+1$ largest spectral radii of $r$-uniform supertrees with size $m$ and diameter $d$. In addition, the first two smallest spectral radii of supertrees with size $m$ are determined.
\end{abstract}

Keywords: Hypergraph; Adjacency tensor; Eigenvalues; Matching polynomial; Supertree

Mathematics Subject Classifications: 15A18, 05C65, 05C31

\footnotetext{
* Research was partially supported by the National Nature Science Foundation of China (grant numbers 11871329, 11561032).

$\dagger$ Corresponding authors.

$\ddagger$ The author was also supported by the Jiangxi Science Fund for Distinguished Young Scholars (No. 20171BCB23032) and the funds of the Education Department of Jiangxi Province (No. GJJ150345).

$\S$ Corresponding authors. The author was supported by the NSFC (No. 11571222).
} 


\section{Introduction}

The ordering of graphs by spectral radius was proposed by Collatz and Sinogowitz [7] in 1957. Lovász and Pelikán [21] investigated the spectral radius of trees and determined the first two largest and smallest spectral radii of trees with given order. Brualdi and Solheid [2] proposed the problem of bounding the spectral radius of some class of graphs and characterizing the corresponding extremal graphs. Since then, many authors studied the spectral radius of trees with some given parameters, such as degree, diameter, etc.

A hypergraph $\mathcal{H}$ is a pair $(V, E)$, where $E \subseteq \mathcal{P}(V)$ and $\mathcal{P}(V)$ stands for the power set of $V$. The elements of $V=V(\mathcal{H})$ are referred to as vertices and the elements of $E=E(\mathcal{H})$ are called hyperedges or edges. A hypergraph $\mathcal{H}$ is $r$-uniform if every edge $e \in E(\mathcal{H})$ contains precisely $r$ vertices. For a vertex $v \in V$, we denote by $E_{v}$ the set of edges containing $v$. The cardinality $\left|E_{v}\right|$ is the degree of $v$, denoted by $\operatorname{deg}(v)$. A vertex with degree one is called a core vertex, and a vertex with degree larger than one is called an intersection vertex. If any two edges in $\mathcal{H}$ share at most one vertex, then $\mathcal{H}$ is said to be a linear hypergraph. In this paper we assume that hypergraphs are $r$-uniform.

In a hypergraph $\mathcal{H}$, two vertices $u$ and $v$ are adjacent if there is an edge $e$ of $\mathcal{H}$ such that $\{u, v\} \subseteq e$. A vertex $v$ is said to be incident to an edge $e$ if $v \in e$. A walk of hypergraph $\mathcal{H}$ is defined to be an alternating sequence of vertices and edges $v_{1} e_{1} v_{2} e_{2} \cdots v_{\ell} e_{\ell} v_{\ell+1}$ satisfying that both $v_{i}$ and $v_{i+1}$ are incident to $e_{i}$ for $1 \leqslant i \leqslant \ell$. A walk is called a path if all vertices and edges in the walk are distinct. The length of a path is the number of edges in it. The walk is closed if $v_{l+1}=v_{1}$. A closed walk is called a cycle if all vertices and edges in the walk are distinct. A hypergraph $\mathcal{H}$ is called connected if any two of its vertices are linked by a path in $\mathcal{H}$. The distance between two vertices is the length of a shortest path connecting them. The diameter of a connected $r$-uniform hypergraph $\mathcal{H}$ is the maximum distance among all vertices of $\mathcal{H}$. A hypergraph $\mathcal{H}$ is called acyclic or a superforest if it contains no cycle. A connected superforest is called a supertree. A supertree is called nontrivial if it has at least one edge.

In [16] some transformations on hypergraphs such as moving edges and edge-releasing were introduced and the first two spectral radii of supertrees on $n$ vertices were characterized. Yuan et al. [33] further determined the first eight uniform supertrees on $n$ vertices with the largest spectral radii. Xiao et al. [27] characterized the unique uniform supertree with the maximum spectral radius among all uniform supertrees with a given degree sequence. Recently, the first two largest spectral radii of uniform supertrees with given diameter were characterized in [28].

In this paper, we determine the first $\left\lfloor\frac{d}{2}\right\rfloor+1$ largest spectral radii of supertrees among all $r$-uniform supertrees with size $m$ and diameter $d$ and the first two smallest spectral radii of supertrees with size $m$. The structure of the remaining part of the paper is as follows: In Section 2, we give some basic definitions and results for tensor and spectra of hypergraphs. Section 3 extends the theory of matching polynomial from graphs to supertrees. By comparing the matching polynomial of supertrees, we generalize Li and Feng's results on grafting operations on graphs to supertrees in Section 4. By using the method of grafting operations on supertrees and comparing matching polynomial of 
supertrees, we determine the first $\left\lfloor\frac{d}{2}\right\rfloor+1$ spectral radii of supertrees among all $r$-uniform supertrees with size $m$ and diameter $d$ in Section 5. In Section 6, the first two smallest spectral radii of supertrees are determined. We give closing remarks in the last section.

\section{Preliminaries}

Let $\mathcal{H}=(V, E)$ be an $r$-uniform hypergraph on $n$ vertices. A partial hypergraph $\mathcal{H}^{\prime}=$ $\left(V^{\prime}, E^{\prime}\right)$ of $\mathcal{H}$ is a hypergraph with $V^{\prime} \subseteq V$ and $E^{\prime} \subseteq E$. A proper partial hypergraph $\mathcal{H}^{\prime}$ of $\mathcal{H}$ is partial hypergraph of $\mathcal{H}$ with $\mathcal{H}^{\prime} \neq \mathcal{H}$. For a vertex subset $S \subset V$, let $\mathcal{H}-S=\left(V^{\prime \prime}, E^{\prime \prime}\right)$ be the partial hypergraph of $\mathcal{H}$ satisfying that $V^{\prime \prime}=V \backslash S$, and for any $e \in E$, if $e \subseteq V^{\prime \prime}$, then $e \in E^{\prime \prime}$. When $S=\{v\}, \mathcal{H}-S$ is simply written as $\mathcal{H}-v$. For an edge $e=\left\{v_{1}, \ldots, v_{t}\right\} \in E(\mathcal{H})$, let $\mathcal{H} \backslash e$ stand for the partial hypergraph of $\mathcal{H}$ obtained by deletion of the edge $e$ from $\mathcal{H}$, i.e. $\mathcal{H} \backslash e=(V, E \backslash\{e\})$, and $\mathcal{H}-V(e)$ stand for the partial hypergraph of $\mathcal{H}-\left\{v_{1}, \ldots, v_{t}\right\}$. Denote by $N_{k}$ the hypergraph consisting of $k$ isolated vertices.

Let $\mathcal{G}$ and $\mathcal{H}$ be two $r$-uniform hypergraphs, and $u$ a vertex of $\mathcal{G}$ and $v$ a vertex of $\mathcal{H}$. Denote by $\mathcal{G} \cdot \mathcal{H}$ the coalescence of $\mathcal{G}$ and $\mathcal{H}$, obtained from $\mathcal{G} \cup \mathcal{H}$ by identifying $u$ of $\mathcal{G}$ and $v$ of $\mathcal{H}$ (as a new vertex $w$ ). That is, $V(\mathcal{G} \cdot \mathcal{H})=V(\mathcal{G}-u) \cup V(\mathcal{H}-v) \cup\{w\}$ and $E(\mathcal{G} \cdot \mathcal{H})=E(\mathcal{G}-u) \cup E(\mathcal{H}-v) \cup\left\{e^{\prime} \mid e^{\prime}=e \backslash\{u\} \cup\{w\}, e \in E_{u}\right\} \cup\left\{e^{\prime} \mid e^{\prime}=\right.$ $\left.e \backslash\{v\} \cup\{w\}, e \in E_{v}\right\}$. $\mathcal{H}$ is also called an attached hypergraph at $w$ of $\mathcal{G} \cdot \mathcal{H}$.

Let $G=(V, E)$ be an ordinary graph. For every $r \geqslant 3$, the $r$ th power of $G$, denoted by $G^{r}$, is an $r$-uniform hypergraph with vertex set $V\left(G^{r}\right)=V \cup\left(\cup_{e \in E}\left\{i_{e, 1}, \ldots, i_{e, r-2}\right\}\right)$ and edge set $E\left(G^{r}\right)=\left\{e \cup\left\{i_{e, 1}, \ldots, i_{e, r-2},\right\} \mid e \in E\right\}$. The $r$ th power of an ordinary tree is called a hypertree (see [13]). Note that all hypertrees are supertrees by the definition. Let $P_{m}$ and $S_{m}$ denote the path and the star with $m$ edges, respectively. The $r$ th power of $P_{m}$ and $S_{m}$, denoted by $P_{m}^{r}$ and $S_{m}^{r}$, are called loose path and hyperstar, respectively.

Let $\mathcal{H}=(V, E)$ be an $r$-uniform hypergraph. An edge $e$ is called a pendent edge if $e$ contains exactly $r-1$ core vertices. If $e$ is not a pendent edge, it is called a non-pendent edge. A path $P=\left(v_{0}, e_{1}, v_{1}, \ldots, v_{p-1}, e_{p}, v_{p}\right)$ of $\mathcal{H}$ is called a pendent path (attached at $\left.v_{0}\right)$, if all of the vertices $v_{1}, \ldots, v_{p-1}$ are of degree two, the vertex $v_{p}$ and all the $r-2$ vertices in the set $e_{i} \backslash\left\{v_{i-1}, v_{i}\right\}$ are core vertices in $\mathcal{H}(i=1, \ldots, p)$.

For positive integers $r$ and $n$, a real tensor $\mathcal{A}=\left(a_{i_{1} i_{2} \cdots i_{r}}\right)$ of order $r$ and dimension $n$ refers to a multidimensional array (also called hypermatrix) with entries $a_{i_{1} i_{2} \cdots i_{r}}$ such that $a_{i_{1} i_{2} \cdots i_{r}} \in \mathbb{R}$ for all $i_{1}, i_{2}, \ldots, i_{r} \in[n]$.

The following product of tensors, defined by Shao [26], is a generalization of the matrix product. Let $\mathcal{A}$ and $\mathcal{B}$ be dimension $n$, order $r \geqslant 2$ and order $k \geqslant 1$ tensors, respectively. Define the product $\mathcal{A B}$ to be the tensor $\mathcal{C}$ of dimension $n$ and order $(r-1)(k-1)+1$ with entries as

$$
c_{i \alpha_{1} \cdots \alpha_{r-1}}=\sum_{i_{2}, \ldots, i_{r}=1}^{n} a_{i i_{2} \cdots i_{r}} b_{i_{2} \alpha_{1}} \cdots b_{i_{r} \alpha_{r-1}}
$$

where $i \in[n], \alpha_{1}, \ldots, \alpha_{r-1} \in[n]^{k-1}$. 
From the above definition, if $x=\left(x_{1}, x_{2}, \ldots, x_{n}\right)^{\mathrm{T}} \in \mathbb{C}^{n}$ is a complex column vector of dimension $n$, then by (1) $\mathcal{A} x$ is a vector in $\mathbb{C}^{n}$ whose $i$ th component is given by

$$
(\mathcal{A} x)_{i}=\sum_{i_{2}, \ldots, i_{r}=1}^{n} a_{i i_{2} \cdots i_{r}} x_{i_{2}} \cdots x_{i_{r}}, \quad \text { for each } i \in[n] .
$$

In 2005, Qi [24] and Lim [18] independently introduced the concepts of tensor eigenvalues and the spectra of tensors.

Let $\mathcal{A}$ be an order $r$ dimension $n$ tensor, $x=\left(x_{1}, x_{2}, \ldots, x_{n}\right)^{\mathrm{T}} \in \mathbb{C}^{n}$ a column vector of dimension $n$. If there exists a number $\lambda \in \mathbb{C}$ and a nonzero vector $x \in \mathbb{C}^{n}$ such that

$$
\mathcal{A} x=\lambda x^{[r-1]},
$$

where $x^{[r-1]}$ is a vector with $i$-th entry $x_{i}^{r-1}$, then $\lambda$ is called an eigenvalue of $\mathcal{A}, x$ is called an eigenvector of $\mathcal{A}$ corresponding to the eigenvalue $\lambda$. The spectral radius of $\mathcal{A}$ is the maximum modulus of the eigenvalues of $\mathcal{A}$.

In 2012, Cooper and Dutle [8] defined the adjacency tensors for $r$-uniform hypergraphs.

Definition 1. ([8]) Let $\mathcal{H}=(V, E)$ be an $r$-uniform hypergraph on $n$ vertices. The adjacency tensor of $\mathcal{H}$ is defined as the order $r$ and dimension $n$ tensor $\mathcal{A}(\mathcal{H})=\left(a_{i_{1} i_{2} \cdots i_{r}}\right)$, whose $\left(i_{1} i_{2} \cdots i_{r}\right)$-entry is

$$
a_{i_{1} i_{2} \cdots i_{r}}= \begin{cases}\frac{1}{(r-1) !}, & \text { if }\left\{i_{1}, i_{2}, \ldots, i_{r}\right\} \in E, \\ 0, & \text { otherwise. }\end{cases}
$$

The spectral radius of hypergraph $\mathcal{H}$ is defined as spectral radius of its adjacency tensor, denoted by $\rho(\mathcal{H})$. In [9] the weak irreducibility of nonnegative tensors was defined. It was proved that an $r$-uniform hypergraph $\mathcal{H}$ is connected if and only if its adjacency tensor $\mathcal{A}(\mathcal{H})$ is weakly irreducible (see [9] and [32]). Part of the Perron-Frobenius theorem for nonnegative tensors is stated in the following for reference.

Theorem 2. ([25]) Let $\mathcal{A}$ be a nonnegative tensor of order $r$ and dimension $n$, where $r, n \geqslant 2$. Then $\rho(\mathcal{A})$ is an eigenvalue of $\mathcal{A}$ with a nonnegative eigenvector corresponding to it. If $\mathcal{A}$ is weakly irreducible, then $\rho(\mathcal{A})$ is a positive eigenvalue of $\mathcal{A}$ with a positive eigenvector $x$. Furthermore, $\rho(\mathcal{A})$ is the unique eigenvalue of $\mathcal{A}$ with a positive eigenvector, and $x$ is the unique positive eigenvector associated with $\rho(\mathcal{A})$, up to a multiplicative constant.

The unique positive eigenvector $x$ with $\sum_{i=1}^{n} x_{i}^{r}=1$ corresponding to $\rho(\mathcal{H})$ is called the principal eigenvector of $\mathcal{H}$.

The following result can be found in $[8,15]$ and will be often used in the sequel.

Theorem 3. ([8],[15]) Suppose that $\mathcal{G}$ is a uniform hypergraph, and $\mathcal{G}^{\prime}$ is a partial hypergraph of $\mathcal{G}$. Then $\rho\left(\mathcal{G}^{\prime}\right) \leqslant \rho(\mathcal{G})$. Furthermore, if in addition $\mathcal{G}$ is connected and $\mathcal{G}^{\prime}$ is a proper partial hypergraph, we have $\rho\left(\mathcal{G}^{\prime}\right)<\rho(\mathcal{G})$. 
An operation of moving edges on hypergraphs was introduced by $\mathrm{Li}$ et al. in [16]. Let $\mathcal{H}=(V, E)$ be a hypergraph with $u \in V$ and $e_{1}, \ldots, e_{k} \in E$, such that $u \notin e_{i}$ for $i=1, \ldots, k$. Suppose that $v_{i} \in e_{i}$ and write $e_{i}^{\prime}=\left(e_{i} \backslash\left\{v_{i}\right\}\right) \cup\{u\}(i=1, \ldots, k)$. Let $\mathcal{H}^{\prime}=\left(V, E^{\prime}\right)$ be the hypergraph with $E^{\prime}=\left(E \backslash\left\{e_{1}, \ldots, e_{k}\right\}\right) \cup\left\{e_{1}^{\prime}, \ldots, e_{k}^{\prime}\right\}$. Then we say that $\mathcal{H}^{\prime}$ is obtained from $\mathcal{H}$ by moving edges $\left(e_{1}, \ldots, e_{k}\right)$ from $\left(v_{1}, \ldots, v_{k}\right)$ to $u$.

Theorem 4. ([16]) Let $\mathcal{H}$ be a connected and uniform hypergraph, $\mathcal{H}^{\prime}$ be the hypergraph obtained from $\mathcal{H}$ by moving edges $\left(e_{1}, \ldots, e_{k}\right)$ from $\left(v_{1}, \ldots, v_{k}\right)$ to $u$, and $\mathcal{H}^{\prime}$ contain no multiple edges. If $x$ is the principal eigenvector of $\mathcal{H}$ corresponding to $\rho(\mathcal{H})$, and suppose that $x_{u} \geqslant \max _{1 \leqslant i \leqslant k}\left\{x_{v_{i}}\right\}$, then $\rho\left(\mathcal{H}^{\prime}\right)>\rho(\mathcal{H})$.

The following edge-releasing operation on linear hypergraphs was given in [16].

Let $\mathcal{H}$ be an $r$-uniform linear hypergraph, $e$ be a non-pendent edge of $\mathcal{H}$ and $u \in e$. Let $e_{1}, e_{2}, \ldots, e_{k}$ be all edges of $G$ adjacent to $e$ but not containing $u$, and suppose that $e_{i} \cap e=\left\{v_{i}\right\}$ for $i=1, \ldots, k$. Let $\mathcal{H}^{\prime}$ be the hypergraph obtained from $\mathcal{H}$ by moving edges $\left(e_{1}, \ldots, e_{k}\right)$ from $\left(v_{1}, \ldots, v_{k}\right)$ to $u$. Then $\mathcal{H}^{\prime}$ is said to be obtained by an edge-releasing operation on $e$ at $u$.

By the above definition we see that if $\mathcal{H}^{\prime}$ and $\mathcal{H}^{\prime \prime}$ are the hypergraphs obtained from an $r$-uniform linear hypergraph $\mathcal{H}$ by an edge-releasing operation on some $e$ at $u$ and at $v$, respectively. Then $\mathcal{H}^{\prime}$ and $\mathcal{H}^{\prime \prime}$ are isomorphic. So we simply say $\mathcal{H}^{\prime}$ is obtained from $\mathcal{H}$ by an edge-releasing operation on $e$.

The following result was obtained by Zhou et al. [35], we will use it in the sequel.

Theorem 5. ([35]) If $\lambda \neq 0$ is an eigenvalue of a graph $G$, then $\lambda^{\frac{2}{r}}$ is an eigenvalue of $G^{r}$. Moreover, $\rho\left(G^{r}\right)=\rho(G)^{\frac{2}{r}}$.

\section{The matching polynomial of hypergraphs}

Let $\mathcal{H}=(V, E)$ be an $r$-uniform hypergraph of order $n$ and size $m$. A matching of $\mathcal{H}$ is a set of pairwise nonadjacent edges in $E$. A $k$-matching is a matching consisting of $k$ edges. We denote by $m(\mathcal{H}, k)$ the number of $k$-matchings of $\mathcal{H}$. The matching number $\nu(\mathcal{H})$ of $\mathcal{H}$ is the maximum cardinality of a matching.

Recently, Zhang et al. [34] obtained the following result.

Theorem 6. ([34]) $\lambda$ is a nonzero eigenvalue of a supertree $\mathcal{H}$ with the corresponding eigenvector $x$ having all elements nonzero if and only if it is a root of the polynomial

$$
\varphi(\mathcal{H}, x)=\sum_{k=0}^{\nu(\mathcal{H})}(-1)^{k} m(\mathcal{H}, k) x^{(\nu(\mathcal{H})-k) r} .
$$

Based on the result above, Clark and Cooper [6] called the polynomial in Theorem 6 as matching polynomial of $\mathcal{H}$. Set $m(\mathcal{H}, 0)=1$. We redefine the matching polynomial of $\mathcal{H}$ as

$$
\varphi(\mathcal{H}, x)=\sum_{k \geqslant 0}(-1)^{k} m(\mathcal{H}, k) x^{n-k r} .
$$


For exmaple, the matching polynomial of $N_{k}$ is $\varphi\left(N_{k}, x\right)=x^{k}$, rather than 1 by Zhang's definition. The definition here seems more appropriate as it guarantees that matching polynomials of hypergraphs of the same order have the same degree and the result in Theorem 6 is still valid.

Some classical results on matching polynomial of a graph can be extended to a hypergraph as well. However, the matching polynomial of a hypergraph has its own flavour, e.g. as shown in [6], the roots of matching polynomial of an $r$-uniform hypergraph with $r>2$ need not necessarily be real.

Theorem 7. Let $\mathcal{G}$ and $\mathcal{H}$ be two r-uniform hypergraphs. Then the following statements hold.

(a) $\varphi(\mathcal{G} \cup \mathcal{H}, x)=\varphi(\mathcal{G}, x) \varphi(\mathcal{H}, x)$.

(b) $\varphi(\mathcal{G}, x)=\varphi(\mathcal{G} \backslash e, x)-\varphi(\mathcal{G}-V(e), x)$ if $e$ is an edge of $\mathcal{G}$.

(c) If $u \in V(\mathcal{G})$ and $I=\left\{i \mid e_{i} \in E_{u}\right\}$, for any $J \subseteq I$, we have

$$
\varphi(\mathcal{G}, x)=\varphi\left(\mathcal{G} \backslash\left\{e_{i}: i \in J\right\}, x\right)-\sum_{i \in J} \varphi\left(\mathcal{G}-V\left(e_{i}\right), x\right)
$$

and

$$
\varphi(\mathcal{G}, x)=x \varphi(\mathcal{G}-u, x)-\sum_{e \in E_{u}} \varphi(\mathcal{G}-V(e), x)
$$

(d) $\sum_{u \in V(\mathcal{G})} \varphi(\mathcal{G}-u, x)=\frac{d}{d x} \varphi(\mathcal{G}, x)$.

Proof. (a) From the fact that each $k$-matching in $\mathcal{G} \cup \mathcal{H}$ consists of an $s$-matching in $\mathcal{G}$ combined with a $(k-s)$-matching from $\mathcal{H}$ for some $s$, the result follows immediately.

(b) In order to compute the matching polynomial, we count the number of $k$-matching in $\mathcal{G}$ according to the edge $e$ being contained or not. The number of $k$-matching not containing $e$ is equal to $m(\mathcal{G} \backslash e, k)$. The number of $k$-matching containing $e$ is equal to $m(\mathcal{G}-V(e), k-1)$. Thus we have

$$
m(\mathcal{G}, k)=m(\mathcal{G} \backslash e, k)+m(\mathcal{G}-V(e), k-1) .
$$

By comparing the coefficients of the corresponding matching polynomial in two sides of (b), the result follows.

(c) Assume that $\left\{e_{i}\right\}_{i \in J}=\left\{e_{1}, \ldots, e_{s}\right\}$. Applying (b) of Theorem 7, we have

$$
\begin{aligned}
\varphi(\mathcal{G}, x) & =\varphi\left(\mathcal{G} \backslash e_{1}, x\right)-\varphi\left(\mathcal{G}-V\left(e_{1}\right), x\right) \\
& =\varphi\left(\mathcal{G} \backslash\left\{e_{1}, e_{2}\right\}, x\right)-\varphi\left(\mathcal{G}-V\left(e_{2}\right), x\right)-\varphi\left(\mathcal{G}-V\left(e_{1}\right), x\right) .
\end{aligned}
$$


Repeatedly using (b) of Theorem 7, we get

$$
\begin{aligned}
\varphi(\mathcal{G}, x) & =\varphi\left(\mathcal{G} \backslash\left\{e_{1}, e_{2}, \ldots, e_{s}\right\}, x\right)-\sum_{i=1}^{s} \varphi\left(\mathcal{G}-V\left(e_{i}\right), x\right) \\
& =\varphi\left(\mathcal{G} \backslash\left\{e_{i}: i \in J\right\}, x\right)-\sum_{i \in J} \varphi\left(\mathcal{G}-V\left(e_{i}\right), x\right) .
\end{aligned}
$$

Note that $u$ is an isolated vertex of $\mathcal{G} \backslash\left\{e_{i}: i \in I\right\}$, it follows directly from (2) that

$$
\varphi(\mathcal{G}, x)=x \varphi(\mathcal{G}-u, x)-\sum_{e_{i} \in E_{u}(G)} \varphi\left(\mathcal{G}-V\left(e_{i}\right), x\right)
$$

(d) Consider the ordered pairs $(u, M)$, where $M$ is a $k$-matching in $\mathcal{G}$ and $u$ is a vertex of $\mathcal{G}$ not covered by $M$. Counting the number of the ordered pairs, we obtain that the number of such ordered pairs is equal to $m(\mathcal{G}, k)(n-r k)$, which is just the absolute value of the coefficient of $x^{n-r k-1}$ in $\frac{d}{d x} \varphi(\mathcal{G}, x)$. On the other hand, if we choose a vertex first, say $u$, then the number of $k$-matching not covering $u$ is equal to $m(\mathcal{G}-u, k)$. Then, the number of such ordered pairs is equal to $\sum_{u \in V(\mathcal{G})} m(\mathcal{G}-u, k)$, which is the absolute value of the coefficient of $x^{n-r k-1}$ in $\sum_{u \in V(\mathcal{G})} \varphi(\mathcal{G}-u, x)$. The desired result follows.

Proposition 8. Let $\mathcal{T}$ be a nontrivial $r$-uniform supertree. Then the spectral radius $\rho(\mathcal{T})$ of $\mathcal{T}$ is a simple root of the matching polynomial $\varphi(\mathcal{T}, x)$ of $\mathcal{T}$.

Proof. First by Theorem 6, $\rho(\mathcal{T})$ is indeed a root of $\varphi(\mathcal{T}, x)$. Suppose, by contradiction, $\rho(\mathcal{T})$ is a multiple root of $\varphi(\mathcal{T}, x)$. Then $\rho(\mathcal{T})$ must be a root of $\frac{d}{d x} \varphi(\mathcal{T}, x)$, that is, $\frac{d}{d x} \varphi(\mathcal{T}, \rho(\mathcal{T}))=0$. However, by (d) of Theorem $7, \frac{d}{d x} \varphi(\mathcal{T}, x)=\sum_{u \in V(\mathcal{T})} \varphi(\mathcal{T}-u, x)$, and for any $u \in V(\mathcal{T}), \varphi(\mathcal{T}-u, \rho(\mathcal{T}))>0$ due to $\rho(\mathcal{T})>\rho(\mathcal{T}-u$ ) by Theorem 3 . Thus $\frac{d}{d x} \varphi(\mathcal{T}, \rho(\mathcal{T}))>0$, a contradiction. Consequently, $\rho(\mathcal{T})$ must be a simple root of $\varphi(\mathcal{T}, x)$.

Proposition 9. Let $T$ be an ordinary tree on $n$ vertices, $r(r \geqslant 3)$ a positive integer. Then the matching polynomials of $T$ and its $r$ th power $T^{r}$ satisfy the following relation:

$$
\varphi\left(T^{r}, x\right)=x^{\frac{(n-2)(r-2)}{2}} \varphi\left(T, x^{\frac{r}{2}}\right) .
$$

Proof. It is easy to see that $m(T, k)=m\left(T^{r}, k\right)$ for any $k$. Let $n^{\prime}$ denote the order of $T^{r}$. Then $n^{\prime}=n+(n-1)(r-2)$. So we have

$$
\begin{aligned}
\varphi\left(T^{r}, x\right) & =\sum_{k \geqslant 0}(-1)^{k} m\left(T^{r}, k\right) x^{n^{\prime}-k r}=\sum_{k \geqslant 0}(-1)^{k} m(T, k)\left(y^{\frac{2}{r}}\right)^{n^{\prime}-k r} \\
& =y^{\frac{2 n^{\prime}}{r}-n} \sum_{k \geqslant 0}(-1)^{k} m(T, k) y^{n-2 k}=x^{\frac{(n-2)(r-2)}{2}} \varphi\left(T, x^{\frac{r}{2}}\right),
\end{aligned}
$$

where a new variable $y=x^{\frac{r}{2}}$ is used in the second and third equations. 
The ordering on forests has been introduced by Lovász and Pelikán in [21]. Now we extend the ordering on forests to superforests. Let $\mathcal{T}$ and $\mathcal{T}^{\prime}$ be superforests of $n$ vertices. We call $\mathcal{T}^{\prime} \preceq \mathcal{T}$ if $\varphi\left(\mathcal{T}^{\prime}, x\right) \geqslant \varphi(\mathcal{T}, x)$ for every $x \geqslant \rho(\mathcal{T})$; call $\mathcal{T}^{\prime} \prec \mathcal{T}$ if $\mathcal{T}^{\prime} \preceq \mathcal{T}$ and the polynomial $\varphi\left(\mathcal{T}^{\prime}, x\right)-\varphi(\mathcal{T}, x)$ does not vanish at the point $x=\rho(\mathcal{T})$. Note that $\mathcal{T}^{\prime} \prec \mathcal{T}$ $\left(\mathcal{T}^{\prime} \preceq \mathcal{T}\right.$, resp.) implies $\rho\left(\mathcal{T}^{\prime}\right)<\rho(\mathcal{T})\left(\rho\left(\mathcal{T}^{\prime}\right) \leqslant \rho(\mathcal{T})\right.$, resp.) by Proposition 8 .

From (a) of Theorem 7 , we have the following observation.

Remark 10. For $r$-uniform superforests $\mathcal{T}, \mathcal{T}^{\prime}, \mathcal{T}_{i}$ and $\mathcal{T}_{i}^{\prime}$, where $i=1,2$,

- if $\mathcal{T}^{\prime} \preceq \mathcal{T}$, then $\mathcal{T}^{\prime} \cup \mathcal{H} \preceq \mathcal{T} \cup \mathcal{H}$ for any $r$-uniform superforest $\mathcal{H}$;

- if for $i=1,2, \mathcal{T}_{i}^{\prime} \prec \mathcal{T}_{i}$, then $\mathcal{T}_{1}^{\prime} \cup \mathcal{T}_{2}^{\prime} \prec \mathcal{T}_{1} \cup \mathcal{T}_{2}$.

\section{Grafting transformations on uniform supertrees}

Li and Feng [17] investigated how the spectral radius change when a certain transformation is applied to the graph, and obtained the following result.

Theorem 11. ([17]) Let $u, v$ be two vertices of $G$ such that $d(u, v)=m$. Let $G(u, v ; p, q)$ denote the graph obtained from $G$ by attaching a path of length $p$ at $u$ and a path of length $q$ at $v$. Then $\rho(G(u, v ; p, q))>\rho(G(u, v ; p+1, q-1))$ under any of the following conditions

1. $m=0, \operatorname{deg}(u) \geqslant 1$, and $p \geqslant q \geqslant 1$;

2. $m=1, \operatorname{deg}(u) \geqslant 2, \operatorname{deg}(v) \geqslant 2$ and $p \geqslant q \geqslant 1$;

3. $m>1, \operatorname{deg}(u) \geqslant 2, \operatorname{deg}(v) \geqslant 2, p-q \geqslant m$ and $q \geqslant 1$.

Since then, the result has been extensively used in spectral perturbation and proved to be efficient in ordering graphs by spectral radius. The result above is proved by comparing characteristic polynomials of graphs. The characteristic polynomial of a hypergraph is complicated and very little is known about it up to now. However the result of Theorem 6 makes it feasible to compare the spectral radii of supertrees by using the matching polynomials of supertrees.

It is known that for any forest, its matching polynomial and characteristic polynomial coincide. Following a similar proof of Lemma 4 in [21], the following result can be obtained.

Proposition 12. If $a+b=c+d$, $a<c \leqslant d$, then $P_{a} \cup P_{b} \prec P_{c} \cup P_{d}$.

Based on Propositions 9 and 12, the corresponding result for hypertree can be easily obtained.

Proposition 13. If $a+b=c+d, a<c \leqslant d, r(r \geqslant 3)$ is an integer, then $P_{a}^{r} \cup P_{b}^{r} \prec P_{c}^{r} \cup P_{d}^{r}$.

Theorem 14. If $\mathcal{T}$ is an uniform supertree, and $\mathcal{T}^{\prime}$ is a proper partial hypergraph of $\mathcal{T}$ with $V\left(\mathcal{T}^{\prime}\right)=V(\mathcal{T})$, then for any $x \geqslant \rho(\mathcal{T}), \varphi(\mathcal{T}, x)<\varphi\left(\mathcal{T}^{\prime}, x\right)$. In particular, $\mathcal{T}^{\prime} \prec \mathcal{T}$. 
Proof. Without loss of generality, we assume that $\mathcal{T}^{\prime}=\mathcal{T} \backslash e$ for some $e$ in $\mathcal{T}$. If $x \geqslant \rho(\mathcal{T})$, then $x>\rho(\mathcal{T}-V(e))$ by Theorem 3. So $\varphi(\mathcal{T}-V(e), x)>0$. Further by Theorem 7 ,

$$
\varphi(\mathcal{T}, x)=\varphi\left(\mathcal{T}^{\prime}, x\right)-\varphi(\mathcal{T}-V(e), x)<\varphi\left(\mathcal{T}^{\prime}, x\right),
$$

the desired result follows.

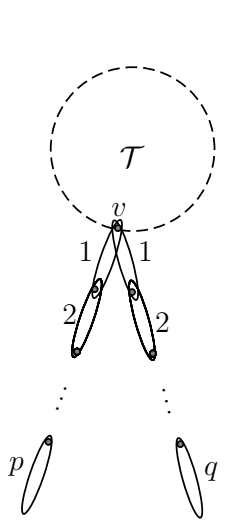

(a)
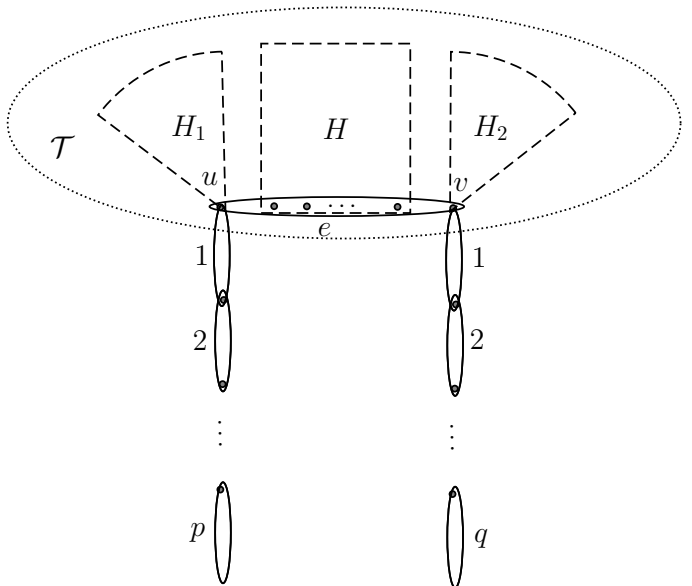

(b)

Figure 1: Supertrees $(a) \mathcal{T}(v ; p, q) ; \quad(b) \mathcal{T}^{(1)}(u, v ; p, q)$.

Suppose that $\mathcal{T}$ is an $r$-uniform supertree and $v$ is a vertex in $\mathcal{T}$. Let $\mathcal{T}(v ; p, q)$ be obtained by attaching two pendent paths of length $p$ and $q$ at $v$ (see Fig. 1(a)).

Theorem 15. If $\mathcal{T}$ is a nontrivial $r$-uniform supertree and $p \geqslant q \geqslant 1$, then $\mathcal{T}(v ; p, q) \succ$ $\mathcal{T}(v ; p+1, q-1)$. In particular, $\rho(\mathcal{T}(v ; p, q))>\rho(\mathcal{T}(v ; p+1, q-1))$.

Proof. We first consider the case that $p \geqslant q=1$. Applying (b) of Theorem 7 on $\mathcal{T}(v ; p, 1)$ and the pendent edge attached at $v$, we have

$$
\varphi(\mathcal{T}(v ; p, 1), x)=x^{r-1} \varphi(\mathcal{T}(v ; p, 0), x)-x^{r-2} \varphi\left((\mathcal{T}-v) \cup P_{p-1}^{r}, x\right) .
$$

Similarly, applying (b) of Theorem 7 on $\mathcal{T}(v ; p+1,0)$ and the pendent edge of the pendent path of length $p+1$ attached at $v$, we have

$$
\varphi(\mathcal{T}(v ; p+1,0), x)=x^{r-1} \varphi(\mathcal{T}(v ; p, 0), x)-x^{r-2} \varphi(\mathcal{T}(v ; p-1,0), x)
$$

By (3) and (4), we deduce that

$$
\varphi(\mathcal{T}(v ; p, 1), x)-\varphi(\mathcal{T}(v ; p+1,0), x)=x^{(r-2)}\left(\varphi(\mathcal{T}(v ; p-1,0), x)-\varphi\left((\mathcal{T}-v) \cup P_{p-1}^{r}, x\right)\right) .
$$

Note that $(\mathcal{T}-v) \cup P_{p-1}^{r}$ is a proper partial hypergraph of $\mathcal{T}(v ; p-1,0)$. By Theorems 3 and 14 , the desired result follows. 
When $p \geqslant q \geqslant 2$, applying (b) of Theorem 7 on $\mathcal{T}(v ; p, q)$ and the pendent edge of the pendent path of length $q$ attached at $v$, we have

$$
\varphi(\mathcal{T}(v ; p, q), x)=x^{r-1} \varphi(\mathcal{T}(v ; p, q-1), x)-x^{r-2} \varphi(\mathcal{T}(v ; p, q-2), x) .
$$

Similarly,

$$
\varphi(\mathcal{T}(v ; p+1, q-1), x)=x^{r-1} \varphi(\mathcal{T}(v ; p, q-1), x)-x^{r-2} \varphi(\mathcal{T}(v ; p-1, q-1), x) .
$$

By (5) and (6), we deduce that

$$
\begin{aligned}
& \varphi(\mathcal{T}(v ; p, q), x)-\varphi(\mathcal{T}(v ; p+1, q-1), x) \\
& =x^{r-2}(\varphi(\mathcal{T}(v ; p-1, q-1), x)-\varphi(\mathcal{T}(v ; p, q-2), x))
\end{aligned}
$$

Continue this process, we get

$$
\begin{aligned}
& \varphi(\mathcal{T}(v ; p, q), x)-\varphi(\mathcal{T}(v ; p+1, q-1), x) \\
= & x^{(r-2)(q-1)}(\varphi(\mathcal{T}(v ; p-q+1,1), x)-\varphi(\mathcal{T}(v ; p-q+2,0), x)) .
\end{aligned}
$$

Applying Theorem 7 once more, we have

$$
\varphi(\mathcal{T}(v ; p-q+1,1), x)=x^{r-1} \varphi(\mathcal{T}(v ; p-q+1,0), x)-x^{r-2} \varphi\left((\mathcal{T}-v) \cup P_{p-q}^{r}, x\right)
$$

and

$$
\varphi(\mathcal{T}(v ; p-q+2,0), x)=x^{r-1} \varphi(\mathcal{T}(v ; p-q+1,0), x)-x^{r-2} \varphi(\mathcal{T}(v ; p-q, 0), x) .
$$

Substituting (8) and (9) into (7), we obtain

$$
\begin{aligned}
& \varphi(\mathcal{T}(v ; p, q), x)-\varphi(\mathcal{T}(v ; p+1, q-1), x) \\
& =x^{q(r-2)}\left(\varphi(\mathcal{T}(v ; p-q, 0), x)-\varphi\left((\mathcal{T}-v) \cup P_{p-q}^{r}, x\right)\right) .
\end{aligned}
$$

Note that $(\mathcal{T}-v) \cup P_{p-q}^{r}$ is a proper partial hypergraph of $\mathcal{T}(v ; p-q, 0)$. Applying Theorems 3 and 14, we get the desired result.

It should be pointed out that the result in Theorem 15 has been proved in [29] for general $r$-uniform hypergraph.

Suppose that $\mathcal{T}$ is an $r$-uniform supertree (with at least two edges) and $u$ and $v$ are two vertices incident with an edge $e$ in $\mathcal{T}$. Let $\mathcal{T}^{(1)}(u, v ; p, q)$ (see Fig. 1(b)) be obtained by attaching two pendent paths of length $p$ and $q$ at $u$ and $v$, respectively.

Theorem 16. Let $\mathcal{T}$ be the supertree defined as above, and further satisfy that one vertex in $e \backslash\{u\}$ is of degree at least 2. If $p \geqslant q \geqslant 1$, then

$$
\mathcal{T}^{(1)}(u, v ; p, q) \succ \mathcal{T}^{(1)}(u, v ; p+1, q-1) .
$$

In particularly,

$$
\rho\left(\mathcal{T}^{(1)}(u, v ; p, q)\right)>\rho\left(\mathcal{T}^{(1)}(u, v ; p+1, q-1)\right)
$$


Proof. Using the similar argument as in the proof of Theorem 15, we have

$$
\begin{aligned}
& \varphi\left(\mathcal{T}^{(1)}(u, v ; p, q), x\right)-\varphi\left(\mathcal{T}^{(1)}(u, v ; p+1, q-1), x\right) \\
& =x^{(r-2)(q-1)}\left(\varphi\left(\mathcal{T}^{(1)}(u, v ; p-q+1,1), x\right)-\varphi\left(\mathcal{T}^{(1)}(u, v ; p-q+2,0), x\right)\right) \\
& =x^{(r-2)(q-1)}\left(x^{r-2} \varphi(\mathcal{T}(u ; p-q, 0), x)-\varphi((\mathcal{T}-v)(u ; p-q+1,0), x)\right) .
\end{aligned}
$$

Let $H_{1}$ and $H_{2}$ be the components of $\mathcal{T} \backslash e$ containing vertex $u$ and $v$ respectively, and $H$ be the union of the remaining components. We denote $H^{\prime}$ as the partial hypergraph of $H$ obtained from $H$ by removing $r-2$ vertices contained in $e$.

When $p=q \geqslant 1$, applying (b) of Theorem 7 to $\mathcal{T}(u ; 0,0)$ and edge $e$, we have

$$
\varphi(\mathcal{T}(u ; 0,0), x)=\varphi\left(H_{1} \cup H \cup H_{2}, x\right)-\varphi\left(\left(H_{1}-u\right) \cup H^{\prime} \cup\left(H_{2}-v\right), x\right) .
$$

Similarly, applying $(\mathrm{b})$ of Theorem 7 to $(\mathcal{T}-v)(u ; 1,0)$ and the pendent edge attached at $u$, we have

$$
\begin{aligned}
& \varphi((\mathcal{T}-v)(u ; 1,0), x) \\
& =x^{r-1} \varphi\left(H_{1} \cup H \cup\left(H_{2}-v\right), x\right)-\varphi\left(\left(H_{1}-u\right) \cup H \cup\left(H_{2}-v\right), x\right) .
\end{aligned}
$$

Substituting (11) and (12) into (10), we obtain

$$
\begin{aligned}
\varphi\left(\mathcal{T}^{(1)}(u, v ; p, q), x\right)-\varphi\left(\mathcal{T}^{(1)}(u, v ; p+1, q-1), x\right) \\
=x^{q(r-2)}\left[\varphi\left(H_{1} \cup H \cup H_{2}, x\right)-x \varphi\left(H_{1} \cup H \cup\left(H_{2}-v\right), x\right)\right] \\
\quad+x^{(q-1)(r-2)}\left[\varphi\left(\left(H_{1}-u\right) \cup H \cup\left(H_{2}-v\right), x\right)-x^{r-2} \varphi\left(\left(H_{1}-u\right) \cup H^{\prime} \cup\left(H_{2}-v\right), x\right)\right] \\
=x^{q(r-2)}\left[\varphi\left(H_{1} \cup H \cup H_{2}, x\right)-\varphi\left(H_{1} \cup H \cup\left(H_{2}-v\right) \cup\{v\}, x\right)\right]+x^{(q-1)(r-2)}\left[\varphi \left(\left(H_{1}-u\right)\right.\right. \\
\left.\left.\quad \cup H \cup\left(H_{2}-v\right), x\right)-\varphi\left(\left(H_{1}-u\right) \cup H^{\prime} \cup(V(e)-\{u, v\}) \cup\left(H_{2}-v\right), x\right)\right] .
\end{aligned}
$$

Observe that $E(H) \cup E\left(H_{2}\right) \neq \varnothing$ follows from the assumption that one vertex in $e \backslash\{u\}$ is of degree at least 2. Since $E(H) \cup E\left(H_{2}\right) \neq \varnothing$, either $\left(H_{2}-v\right) \cup\{v\}$ is a proper partial hypergraph of $H_{2}$, or $H^{\prime} \cup(V(e)-\{u, v\})$ is a proper partial hypergraph of $H$. By Theorems 3, 14 and (13), the result follows.

When $p>q \geqslant 1$, applying (b) of Theorem 7 to $\mathcal{T}(u ; p-q, 0)$ and the edge $e$, we have

$$
\begin{aligned}
\varphi(\mathcal{T}(u ; p-q, 0), x)= & \varphi\left(H_{1}(u ; p-q, 0) \cup H \cup H_{2}\right) \\
& -x^{r-2} \varphi\left(\left(H_{1}-u\right) \cup P_{p-q-1}^{r} \cup H^{\prime} \cup\left(H_{2}-v\right)\right) .
\end{aligned}
$$

Similarly, applying (b) of Theorem 7 to $(\mathcal{T}-v)(u ; p-q+1,0)$ and the pendent edge of the pendent path of length $p-q+1$ attached at $u$, we have

$$
\begin{aligned}
\varphi((\mathcal{T}-v)(u ; p-q+1,0), x)= & x^{r-1} \varphi\left(H_{1}(u ; p-q, 0) \cup H \cup\left(H_{2}-v\right), x\right) \\
& -x^{r-2} \varphi\left(H_{1}(u ; p-q-1,0) \cup H \cup\left(H_{2}-v\right), x\right) .
\end{aligned}
$$


Substituting (14) and (15) into (10) yields

$$
\begin{aligned}
& \varphi\left(\mathcal{T}^{(1)}(u, v ; p, q), x\right)-\varphi\left(\mathcal{T}^{(1)}(u, v ; p+1, q-1), x\right) \\
& \left.=x^{q(r-1)} \varphi\left(H_{1}(u ; p-q, 0), x\right) \varphi(H, x)\left[\varphi\left(H_{2}, x\right)-x \varphi\left(H_{2}-v\right), x\right)\right] \\
& +x^{q(r-1)} \varphi\left(H_{2}-v, x\right)\left[\varphi\left(H_{1}(u ; p-q-1,0) \cup H, x\right)-\varphi\left(\left(H_{1}-u\right)\right.\right. \\
& \left.\left.\cup P_{p-q-1}^{r} \cup H^{\prime} \cup N_{r-2}, x\right)\right] .
\end{aligned}
$$

We consider the following two cases depending on whether or not $E\left(H_{1}\right) \cup E\left(H_{2}\right)$ is empty.

Case 1. $E\left(H_{1}\right) \cup E\left(H_{2}\right) \neq \varnothing$. Without loss of generality, we assume that $E\left(H_{1}\right) \neq \varnothing$. It is easily seen that $\left(H_{1}-u\right) \cup P_{p-q-1}^{r}$ is a proper partial hypergraph of $H_{1}(u ; p-q-1,0)$. By Theorems 3, 14 and (16), we prove the desired result.

Case 2. $E\left(H_{1}\right) \cup E\left(H_{2}\right)=\varnothing$. Since $E\left(H_{1}\right)$ is empty, $H_{1}(u ; p-q-1,0)$ and $\left(H_{1}-u\right) \cup P_{p-q-1}^{r}$ are equal to $P_{p-q-1}^{r}$. Since $\left.E\left(H_{2}\right)=\varnothing, \varphi\left(H_{2}, x\right)-x \varphi\left(H_{2}-v\right), x\right)=0$. Then (16) becomes

$$
\begin{aligned}
& \varphi\left(\mathcal{T}^{(1)}(u, v ; p, q), x\right)-\varphi\left(\mathcal{T}^{(1)}(u, v ; p+1, q-1), x\right) \\
& =x^{q(r-1)} \varphi\left(H_{2}-v, x\right) \varphi\left(P_{p-q-1}^{r}, x\right)\left[\varphi(H, x)-\varphi\left(H^{\prime} \cup N_{r-2}, x\right)\right] .
\end{aligned}
$$

Note that $E(H) \neq \varnothing$ in this case. So $H^{\prime} \cup N_{r-2}$ is proper partial hypergraph of $H$. By Theorems 3, 14 and (16), desired result follows.

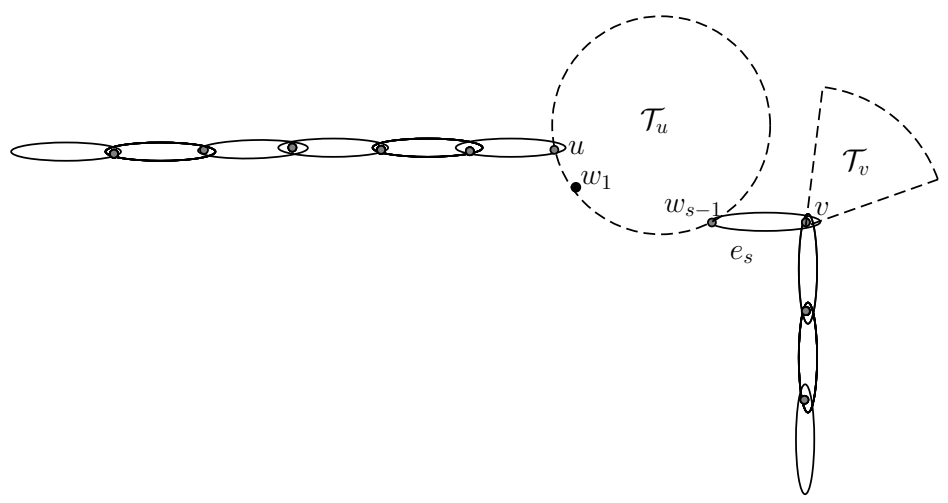

Figure 2: Supertree $\mathcal{T}^{(s)}(u, v ; 6,3)$.

Suppose that $\mathcal{T}$ is an $r$-uniform supertree and $u$ and $v$ are two vertices connected by a path $P$ of length $s$ in $\mathcal{T}$, say $P=\left(u, e_{1}, w_{1}, e_{2}, w_{2}, \ldots, e_{s-1}, w_{s-1}, e_{s}, v\right)$, and all the $r-2$ vertices in the set $e_{i} \backslash\left\{w_{i-1}, w_{i}\right\}$ are of degree one in $\mathcal{T}$ for $i=2, \ldots, s$, where $w_{s}=v$. Let $\mathcal{T}^{(s)}(u, v ; p, q)$ be obtained by attaching two pendent paths of length $p$ and $q$ at $u$ and $v$ respectively (see Fig. 2). 
Theorem 17. Let $\mathcal{T}$ be the supertree defined as above, and further satisfy that $P$ is not a pendent path attached at $u$. If $p-q \geqslant s \geqslant 1$ and $q \geqslant 1$, then

$$
\mathcal{T}^{(s)}(u, v ; p, q) \succ \mathcal{T}^{(s)}(u, v ; p+1, q-1) .
$$

In particular,

$$
\rho\left(\mathcal{T}^{(s)}(u, v ; p, q)\right)>\rho\left(\mathcal{T}^{(s)}(u, v ; p+1, q-1)\right) .
$$

Proof. We proceed by induction on $s$. For the case $s=1$, the assertion holds by Theorem 16. Let $\mathcal{T}_{u}$ and $\mathcal{T}_{v}$ denote the components of $\mathcal{T} \backslash e_{s}$ containing $u$ and $v$, respectively. Using the similar argument as in the proof of Theorem 16, we have

$$
\begin{aligned}
& \varphi\left(\mathcal{T}^{(s)}(u, v ; p, q), x\right)-\varphi\left(\mathcal{T}^{(s)}(u, v ; p+1, q-1), x\right) \\
& =x^{(q-1)(r-2)}\left[\varphi\left(\mathcal{T}^{(s)}(u, v ; p-q+1,1), x\right)-\varphi(\mathcal{T}(u ; p-q+2,0), x)\right] \\
& =x^{(q-1)(r-2)}\left[x^{r-2} \varphi(\mathcal{T}(u ; p-q, 0), x)-\varphi((\mathcal{T}-v)(u ; p-q+1,0), x)\right] \\
& =x^{q(r-2)}\left[\varphi(\mathcal{T}(u ; p-q, 0), x)-\varphi\left(\mathcal{T}_{u}^{(s-1)}\left(u, w_{s-1} ; p-q+1,0\right) \cup\left(\mathcal{T}_{v}-v\right), x\right)\right],
\end{aligned}
$$

where the last equality follows from $(\mathcal{T}-v)(u ; p-q+1,0) \cong \mathcal{T}_{u}^{(s-1)}\left(u, w_{s-1} ; p-q+1,0\right) \cup$ $\left(\mathcal{T}_{v}-v\right) \cup N_{r-2}$.

Applying (c) of Theorem 7 to $\mathcal{T}(u ; p-q, 0)$ and the edges incident to $v$ in $\mathcal{T}_{v}$, we have

$$
\begin{aligned}
\varphi(\mathcal{T}(u ; p-q, 0), x)= & \varphi\left(\mathcal{T}_{v}-v, x\right) \varphi\left(\mathcal{T}_{u}^{(s-1)}\left(u, w_{s-1} ; p-q, 1\right), x\right)- \\
& x^{r-2} \varphi\left(\mathcal{T}_{u}(u ; p-q, 0), x\right) \sum_{e \in E_{v} \cap E\left(\mathcal{T}_{v}\right)} \varphi\left(\mathcal{T}_{v}-V(e), x\right) .
\end{aligned}
$$

Substituting (18) into (17), we obtain

$$
\begin{aligned}
\varphi & \left(\mathcal{T}^{(s)}(u, v ; p, q), x\right)-\varphi\left(\mathcal{T}^{(s)}(u, v ; p+1, q-1), x\right) \\
= & x^{q(r-2)} \varphi\left(\mathcal{T}_{v}-v, x\right)\left[\varphi\left(\mathcal{T}_{u}^{(s-1)}\left(u, w_{s-1} ; p-q, 1\right), x\right)-\varphi\left(\mathcal{T}_{u}^{(s-1)}\left(u, w_{s-1} ; p-q+1,0\right), x\right)\right] \\
& -x^{(q+1)(r-2)} \varphi\left(\mathcal{T}_{u}(u ; p-q, 0), x\right) \sum_{e \in E_{v} \cap E\left(\mathcal{T}_{v}\right)} \varphi\left(\mathcal{T}_{v}-V(e), x\right) .
\end{aligned}
$$

By induction hypothesis, $\mathcal{T}_{u}^{(s-1)}\left(u, w_{s-1} ; p-q, 1\right) \succ \mathcal{T}_{u}^{(s-1)}\left(u, w_{s-1} ; p-q+1,0\right)$. Combining this with Theorems 3 and 14, we prove the theorem.

Lemma 18. Let $\mathcal{T}$ be an $r$-uniform supertree with $e$ as a non-pendent edge, and $\mathcal{T}^{\prime}$ be obtained by edge-releasing e of $\mathcal{T}$. Then $\mathcal{T}^{\prime}$ is a uniform supertree and $\mathcal{T} \prec \mathcal{T}^{\prime}$.

Proof. That $\mathcal{T}^{\prime}$ is a uniform supertree has been proved in [16]. $\mathcal{T}$ may be regarded as one consisting of $s \geqslant 2$ supertrees, say $H_{1}, \ldots, H_{s}$, attached at vertices $v_{1}, \ldots, v_{s}$ of $e$, respectively. It suffices to prove the assertion for $s=2$. Let $H_{1} \cdot H_{2}$ be the coalescence of 
$H_{1}$ and $H_{2}$ obtained by identifying $v_{1}$ of $H_{1}$ and $v_{2}$ of $H_{2}$. It is not difficult to verify that $\mathcal{T}^{\prime} \backslash e \cong H_{1} \cdot H_{2} \cup N_{r-1}$ and $\mathcal{T}^{\prime}-V(e) \cong\left(H_{1}-v_{1}\right) \cup\left(H_{2}-v_{2}\right)$. By Theorem 7 , we have

$$
\begin{aligned}
\varphi(\mathcal{T}, x) & =\varphi(\mathcal{T} \backslash e, x)-\varphi(\mathcal{T}-V(e), x) \\
& =x^{r-2} \varphi\left(H_{1} \cup H_{2}, x\right)-\varphi\left(\left(H_{1}-v_{1}\right) \cup\left(H_{2}-v_{2}\right), x\right)
\end{aligned}
$$

and

$$
\begin{aligned}
\varphi\left(\mathcal{T}^{\prime}, x\right) & =\varphi\left(\mathcal{T}^{\prime} \backslash e, x\right)-\varphi\left(\mathcal{T}^{\prime}-V(e), x\right) \\
& =x^{r-1} \varphi\left(H_{1} \cdot H_{2}, x\right)-\varphi\left(\left(H_{1}-v_{1}\right) \cup\left(H_{2}-v_{2}\right), x\right) .
\end{aligned}
$$

By (20) and (21), we deduce that

$$
\varphi(\mathcal{T}, x)-\varphi\left(\mathcal{T}^{\prime}, x\right)=x^{r-2}\left[\varphi\left(H_{1} \cup H_{2}, x\right)-x \varphi\left(H_{1} \cdot H_{2}, x\right)\right] .
$$

Applying (c) of Theorem 7 to $H_{1} \cup H_{2}$ and edges in $H_{1}$ incident to $v_{1}$, we have

$$
\varphi\left(H_{1} \cup H_{2}, x\right)=x \varphi\left(\left(H_{1}-v_{1}\right) \cup H_{2}, x\right)-\varphi\left(H_{2}, x\right) \sum_{e_{i} \in E_{v_{1}} \cap E\left(H_{1}\right)} \varphi\left(H_{1}-V\left(e_{i}\right), x\right) .
$$

Similarly,

$$
\varphi\left(H_{1} \cdot H_{2}, x\right)=\varphi\left(\left(H_{1}-v_{1}\right) \cup H_{2}, x\right)-\varphi\left(H_{2}-v_{2}, x\right) \sum_{e_{i} \in E_{v_{1}} \cap E\left(H_{1}\right)} \varphi\left(H_{1}-V\left(e_{i}\right), x\right) .
$$

Substituting (23) and (24) into (22), we obtain

$$
\varphi(\mathcal{T}, x)-\varphi\left(\mathcal{T}^{\prime}, x\right)=x^{r-2} \sum_{e_{i} \in E_{v_{1}} \cap E\left(H_{1}\right)} \varphi\left(H_{1}-V\left(e_{i}\right), x\right)\left[x \varphi\left(H_{2}-v_{2}, x\right)-\varphi\left(H_{2}, x\right)\right] .
$$

By Theorems 3 and 14 , we have $\varphi(\mathcal{T}, x)-\varphi\left(\mathcal{T}^{\prime}, x\right)>0$ if $x \geqslant \rho\left(\mathcal{T}^{\prime}\right)$, so $\mathcal{T} \prec \mathcal{T}^{\prime}$ holds.

As an application of Theorems 15 and 16, the minimal supertree can be characterized as follows. Note that the upper bound and the extremal supertree have been obtained in [16], and they are listed here for completeness.

Theorem 19. ([16]) If $\mathcal{T}$ is an $r$-uniform supertree with $m$ edges, then

$$
P_{m}^{r} \preceq \mathcal{T} \preceq S_{m}^{r}
$$

and

$$
\left(2 \cos \frac{\pi}{m+2}\right)^{2 / r} \leqslant \rho(\mathcal{T}) \leqslant m^{1 / r}
$$

with left equality in Eq. (25) and Eq. (26) if and only if $\mathcal{T} \cong P_{m}^{r}$ and right equality in Eq. (25) and Eq. (26) if and only if $\mathcal{T} \cong S_{m}^{r}$. 


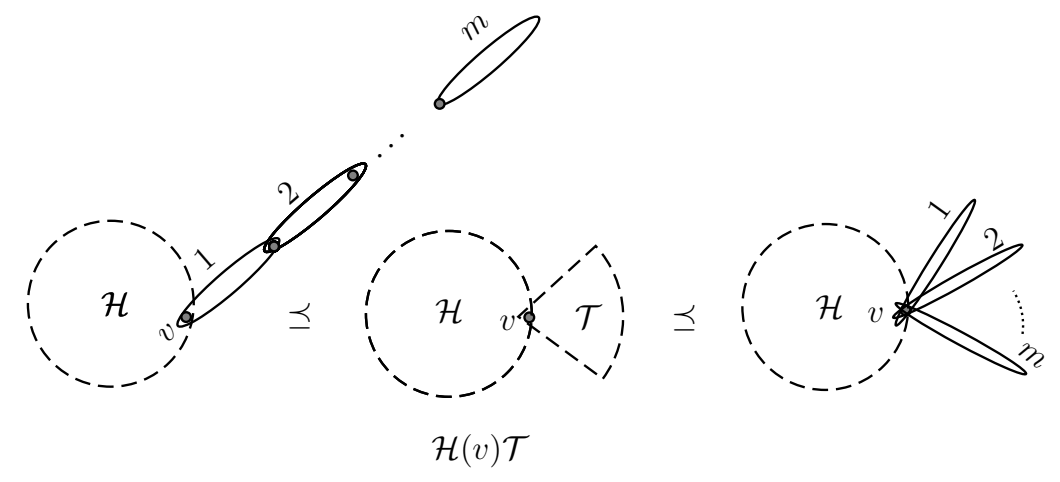

Figure 3: Supertree $\mathcal{H}(v) \mathcal{T}, \mathcal{T}$ with $m$ edges.

Actually, using Theorems 15, 16 and Lemma 18, we can deduce the following more general result.

Theorem 20. Let $\mathcal{H}$ be an $r$-uniform supertree, and $v$ a non-isolated vertex of $\mathcal{H}$. Let $\mathcal{H}(v) \mathcal{T}$ denote the supertree obtained from $\mathcal{H}$ together with an attached supertree $\mathcal{T}$ at $v$ of $\mathcal{H}$, see Fig. 3. Then

$$
\mathcal{H}(v) P_{m}^{r} \preceq \mathcal{H}(v) \mathcal{T} \preceq \mathcal{H}(v) S_{m}^{r},
$$

where the left-hand side equality holds if and only if $\mathcal{T} \cong P_{m}^{r}$ with $v$ as its end vertex whereas the right-hand side equality holds if and only if $\mathcal{T} \cong S_{m}^{r}$ with $v$ as its center.

\section{Extremal supertrees with given diameter}

Let $S(m, d, r)$ be the set of $r$-uniform supertrees with $m$ edges and diameter $d$. Xiao et al. [28] determined the first two largest spectral radii of supertrees in $S(m, d, r)$. In this section, we determine the first $\left\lfloor\frac{d}{2}\right\rfloor+1$ largest spectral radii of supertrees in $S(m, d, r)$ by using edge-grafting operations and comparing matching polynomials of supertrees.

Let $\mathcal{H}$ be an $r$-uniform hypergraph and $u$ a vertex of $\mathcal{H}$. Let $P_{d}^{r}=\left(v_{1}, e_{1}, v_{2}, e_{2}, \ldots, e_{d}\right.$, $\left.v_{d+1}\right)$ be a loose path of length $d$. Denote by $P_{d}^{r}\left(v_{i}, u\right) \mathcal{H}$ and $P_{d}^{r}\left(e_{j}, u\right) \mathcal{H}$ the hypergraphs obtained by identifying vertex $u$ of $\mathcal{H}$ with vertex $v_{i}$ of $P_{d}^{r}$ and a core vertex of $P_{d}^{r}$ in $e_{j}$ respectively (see Fig. 4).

As an immediate application of Theorems 15 and 16, we have the following result.

Theorem 21. Let $\mathcal{T}$ be an $r$-uniform supertree, $r \geqslant 3$. Then

(a) $P_{d}^{r}\left(v_{i}, u\right) \mathcal{T} \succ P_{d}^{r}\left(v_{j}, u\right) \mathcal{T}$, if $2 \leqslant j<i \leqslant\lfloor d / 2\rfloor+1$;

(b) $P_{d}^{r}\left(e_{i}, u\right) \mathcal{T} \succ P_{d}^{r}\left(e_{j}, u\right) \mathcal{T}$, if $2 \leqslant j<i \leqslant\lceil d / 2\rceil$.

Proof. Note that $P_{d}^{r}\left(v_{i}, u\right) \mathcal{T}$ and $P_{d}^{r}\left(e_{i}, u\right) \mathcal{T}$ can be depicted as $\mathcal{T}(u ; i-1, d-i+1)$ and $\left(\mathcal{T}^{\prime}\right)^{(1)}\left(v_{i}, v_{i+1} ; i-1, d-i\right)$ respectively, where $\mathcal{T}^{\prime}$ denotes the supertree consists of $\mathcal{T}$ and $e_{i}$. The assertions follow directly from Theorem 15 and Theorem 16 respectively. 
(a)

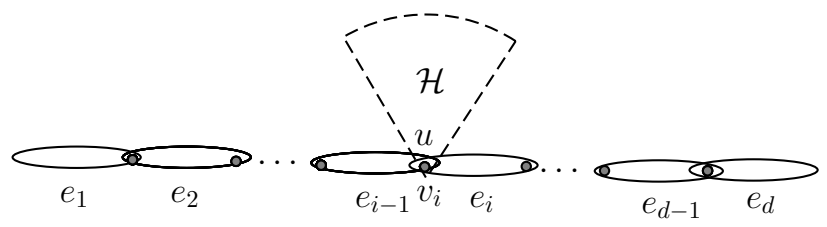

(b)

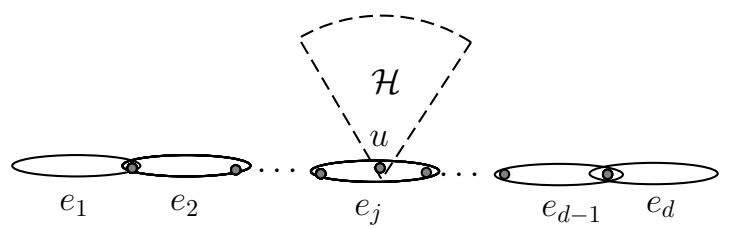

Figure 4: Supertrees (a) $P_{d}^{r}\left(v_{i}, u\right) \mathcal{H} ;(\mathrm{b}) P_{d}^{r}\left(e_{j}, u\right) \mathcal{H}$.

Theorem 22. Let $\mathcal{T}$ be an $r$-uniform supertree and $P_{d}^{r}$ be a loose path of length $d$, with $d \geqslant 3$ and $r \geqslant 3$. Then for any $2 \leqslant i \leqslant d$, we have

$$
P_{d}^{r}\left(e_{\left\lceil\frac{d}{2}\right\rceil}, u\right) \mathcal{T} \prec P_{d}^{r}\left(v_{i}, u\right) \mathcal{T} .
$$

Proof. Suppose that $e_{1}, e_{2}, \ldots, e_{s}$ are all edges incident with vertex $u$ in $\mathcal{T}$. Applying (c) of Theorem 7 to $P_{d}^{r}\left(e_{\left\lceil\frac{d}{2}\right\rceil}, u\right) \mathcal{T}$ and edges $e_{1}, e_{2}, \ldots, e_{s}$, we have

$$
\varphi\left(P_{d}^{r}\left(e_{\left\lceil\frac{d}{2}\right\rceil}, u\right) \mathcal{T}, x\right)=\varphi\left(P_{d}^{r}, x\right) \varphi(\mathcal{T}-u, x)-x^{r-3} \varphi\left(P_{\left\lfloor\frac{d}{2}\right\rfloor}^{r}, x\right) \varphi\left(P_{\left\lceil\frac{d}{2}\right\rceil-1}^{r}, x\right) \sum_{j=1}^{s} \varphi\left(\mathcal{T}-V\left(e_{j}\right), x\right) .
$$

Similarly,

$$
\varphi\left(P_{d}^{r}\left(v_{i}, u\right) \mathcal{T}, x\right)=\varphi\left(P_{d}^{r}, x\right) \varphi(\mathcal{T}-u, x)-x^{2 r-4} \varphi\left(P_{i-2}^{r}, x\right) \varphi\left(P_{d-i}^{r}, x\right) \sum_{j=1}^{s} \varphi\left(\mathcal{T}-V\left(e_{j}\right), x\right) .
$$

Then

$$
\begin{aligned}
& \varphi\left(P_{d}^{r}\left(e_{\left\lceil\frac{d}{2}\right\rceil}, u\right) \mathcal{T}, x\right)-\varphi\left(P_{d}^{r}\left(v_{i}, u\right) \mathcal{T}, x\right) \\
& =x^{r-3} \sum_{j=1}^{s} \varphi\left(\mathcal{T}-V\left(e_{j}\right), x\right)\left[x^{r-1} \varphi\left(P_{i-2}^{r} \cup P_{d-i}^{r}, x\right)-\varphi\left(P_{\left\lfloor\frac{d}{2}\right\rfloor}^{r} \cup P_{\left\lceil\frac{d}{2}\right\rceil-1}^{r}, x\right)\right] .
\end{aligned}
$$

Since

$$
\begin{aligned}
& x^{r-1} \varphi\left(P_{i-2}^{r} \cup P_{d-i}^{r}, x\right)-\varphi\left(P_{\left\lfloor\frac{d}{2}\right\rfloor}^{r} \cup P_{\left\lceil\frac{d}{2}\right\rceil-1}^{r}, x\right) \\
& =\varphi\left(P_{d-i}^{r}, x\right)\left[\varphi\left(P_{i-2}^{r} \cup N_{r-1}, x\right)-\varphi\left(P_{i-1}^{r}, x\right)\right] \\
& \quad+\varphi\left(P_{i-1}^{r} \cup P_{d-i}^{r}, x\right)-\varphi\left(P_{\left\lfloor\frac{d}{2}\right\rfloor}^{r} \cup P_{\left\lceil\frac{d}{2}\right\rceil-1}^{r}, x\right),
\end{aligned}
$$

and $P_{i-1}^{r} \cup P_{d-i}^{r} \preceq P_{\left\lfloor\frac{d}{2}\right\rfloor}^{r} \cup P_{\left\lceil\frac{d}{2}\right\rceil-1}^{r}$ by Proposition 13, we have if $x \geqslant \rho\left(P_{d}^{r}\left(v_{i}, u\right) \mathcal{T}\right)$, then by Theorems 3, 14, $\varphi\left(P_{i-2}^{r} \cup N_{r-1}, x\right)-\varphi\left(P_{i-1}^{r}, x\right)>0$ and $\varphi\left(P_{i-1}^{r} \cup P_{d-i}^{r}, x\right)-\varphi\left(P_{\left\lfloor\frac{d}{2}\right\rfloor}^{r} \cup\right.$ 
$\left.P_{\left\lceil\frac{d}{2}\right\rceil-1}^{r}, x\right) \geqslant 0$. Thus by $(27)$, we have $\varphi\left(P_{d}^{r}\left(e_{\left\lceil\frac{d}{2}\right\rceil}, u\right) \mathcal{T}, x\right)-\varphi\left(P_{d}^{r}\left(v_{i}, u\right) \mathcal{T}, x\right)>0$ if $x \geqslant$ $\rho\left(P_{d}^{r}\left(v_{i}, u\right) \mathcal{T}\right)$. The proof is finished.

The following result follows as an immediate consequence of the above two theorems.

Corollary 23. Let $\mathcal{T}$ be an $r$-uniform supertree, $r \geqslant 3$. Then

(a) $P_{d}^{r}\left(e_{i}, u\right) \mathcal{T} \prec P_{d}^{r}\left(v_{i}, u\right) \mathcal{T}$, if $i=2,3, \ldots, d$;

(b) $P_{d}^{r}\left(e_{i}, u\right) \mathcal{T} \prec P_{d}^{r}\left(v_{i+1}, u\right) \mathcal{T}$, if $i=2,3, \ldots, d-1$.

For convenience, we adopt the notation from [10]. Let $m, d, i$ be integers with $2 \leqslant$ $i \leqslant d \leqslant m-1$, and $T_{m, d}$ be the set of trees of size $m$ and diameter $d$. We use $P=$ $\left(v_{1}, e_{1}, v_{2}, e_{2}, \ldots, v_{d}, e_{d}, v_{d+1}\right)$ to denote the path of length $d$.

Let $T_{(m, d)}(i)$ be the tree on $m$ edges (with diameter $d$ ) obtained from the path $P$ by attaching $m-d$ new pendent edges to the vertex $v_{i}$. Let $T_{(m, d)}=\left\{T_{(m, d)}(i): i=\right.$ $2,3, \ldots, d\}$.

Let $m, d, i, j$ be integers with $2 \leqslant i \neq j \leqslant d \leqslant m-2$. Let $T_{(m, d)}(i, j)$ be the tree on $m$ edges (with diameter $d$ ) obtained from the path $P$ by attaching $m-d-1$ new pendent edges to the vertex $v_{i}$ and a new pendent edge to $v_{j}$, respectively. Let $T^{\prime \prime}=T_{(m, d)}\left(\left\lceil\frac{d}{2}\right\rceil,\left\lceil\frac{d}{2}\right\rceil+1\right)$.

Lemma 24. ([10]) For any tree $T \in T_{m, d} \backslash\left\{\dot{T}_{(m, d)} \cup T^{\prime \prime}\right\}$ with $m \geqslant d+3 \geqslant 6$, we have $\rho(T)<\rho\left(T^{\prime \prime}\right)$.

The following results were obtained in [10] and we shall extend these results from trees to supertrees in this section.

Theorem 25. ([10]) (a) The first $\left\lfloor\frac{d}{2}\right\rfloor+1$ spectral radii of trees in the set $T_{(m, d)}$ with $m \geqslant d+3$ and $d \geqslant 3$ are $T_{(m, d)}\left(\left\lfloor\frac{d}{2}\right\rfloor+1\right), T_{(m, d)}\left(\left\lfloor\frac{d}{2}\right\rfloor\right), \ldots, T_{(m, d)}(3), T_{(m, d)}(2), T^{\prime \prime}$.

(b) The first $\left\lfloor\frac{d}{2}\right\rfloor-1$ spectral radii of trees in the set $T_{(m, d)}$ with $m=d+2$ and $d \geqslant 4$ are $T_{(m, d)}\left(\left\lfloor\frac{d}{2}\right\rfloor+1\right), T_{(m, d)}\left(\left\lfloor\frac{d}{2}\right\rfloor\right), \ldots, T_{(m, d)}(3)$.

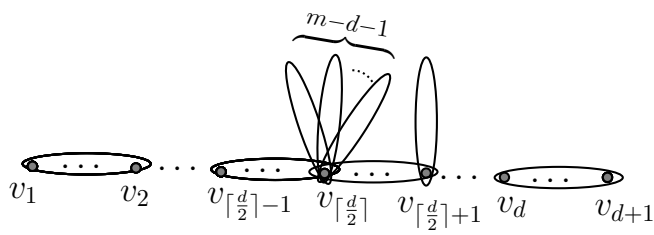

Figure 5: Supertree $\mathcal{T}^{\prime \prime}=T_{(m, d)}^{r}(\lceil d / 2\rceil,\lceil d / 2\rceil+1)$.

Let $m, d, i$ be integers with $2 \leqslant i \leqslant d-1 \leqslant m$. Let $T_{(m, d, r)}(i)$ be a supertree $P_{d}^{r}\left(e_{i}, u\right) \mathcal{T}$, where $T$ is a hyperstar with $m-d$ edges and $u$ as its center. Note that $T_{(m, d, r)}(i) \cong T_{(m, d, r)}(d-i+1)(2 \leqslant i \leqslant d-1)$.

Let $\hat{T}_{(m, d)}^{r}=\left\{T_{(m, d)}^{r}(i): i=2,3, \ldots, d\right\}$ consisting of the $r$ th power of $T_{(m, d)}(i)$ for $i=2,3, \ldots, d$, and let $\mathcal{T}^{\prime \prime}:=T_{(m, d)}^{r}\left(\left\lceil\frac{d}{2}\right\rceil,\left\lceil\frac{d}{2}\right\rceil+1\right)$ (see Fig. 5). 
Lemma 26. For any $m \geqslant d+2 \geqslant 5$ and $r \geqslant 3$, we have $\mathcal{T}^{\prime \prime} \succ T_{(m, d, r)}(\lceil d / 2\rceil)$.

Proof. For simplicity, let $a=m-d-1$ and $b=\left\lceil\frac{d}{2}\right\rceil$. Applying (c) of Theorem 7 to $\mathcal{T}^{\prime \prime}$ and $m-d-1$ pendent edges attached at $v_{\left\lceil\frac{d}{2}\right\rceil}$, we have

$$
\varphi\left(\mathcal{T}^{\prime \prime}, x\right)=x^{a(r-1)} \varphi\left(T_{(d+1, d)}^{r}(b+1), x\right)-a x^{(a+1)(r-1)-2} \varphi\left(P_{b-2}^{r} \cup P_{d-b+1}^{r}, x\right) .
$$

Applying (c) of Theorem 7 to $T_{(d+1, d)}^{r}(b+1)$ and the pendent edge attached at $v_{\left\lceil\frac{d}{2}\right\rceil+1}$, we get

$$
\varphi\left(T_{(d+1, d)}^{r}(b+1), x\right)=x^{r-1} \varphi\left(P_{d}^{r}, x\right)-x^{2(r-2)} \varphi\left(P_{b-1}^{r} \cup P_{d-b-1}^{r}, x\right)
$$

Substituting (29) into (28), we deduce

$$
\begin{aligned}
\varphi\left(\mathcal{T}^{\prime \prime}, x\right) & =x^{a(r-1)} \varphi\left(T_{(d+1, d)}^{r}(b+1), x\right)-a x^{(a+1)(r-1)-2} \varphi\left(P_{b-2}^{r} \cup P_{d-b+1}^{r}, x\right) \\
& =x^{(a+1)(r-1)} \varphi\left(P_{d}^{r}, x\right)-x^{(a+2)(r-1)-2} \varphi\left(P_{b-1}^{r} \cup P_{d-b-1}^{r}, x\right) \\
& -a x^{(a+1)(r-1)-2} \varphi\left(P_{b-2}^{r} \cup P_{d-b+1}^{r}, x\right) .
\end{aligned}
$$

Similarly,

$$
\varphi\left(T_{(m, d, r)}(\lceil d / 2\rceil), x\right)=x^{(a+1)(r-1)} \varphi\left(P_{d}^{r}, x\right)-(a+1) x^{(a+1)(r-1)-2} \varphi\left(P_{b-1}^{r} \cup P_{d-b}^{r}, x\right) .
$$

By (30) and (31), we have

$$
\begin{aligned}
& \varphi\left(\mathcal{T}^{\prime \prime}, x\right)-\varphi\left(T_{(m, d, r)}(\lceil d / 2\rceil), x\right) \\
& =x^{(a+1)(r-1)-2} \varphi\left(P_{b-1}^{r}, x\right)\left[\varphi\left(P_{d-b}^{r}, x\right)-\varphi\left(P_{d-b-1}^{r} \cup N_{r-1}, x\right)\right] \\
& +a x^{(a+1)(r-1)-2}\left[\varphi\left(P_{b-1}^{r} \cup P_{d-b}^{r}, x\right)-\varphi\left(P_{b-2}^{r} \cup P_{d-b+1}^{r}, x\right)\right] .
\end{aligned}
$$

Obviously, $P_{d-b}^{r} \succ P_{d-b-1}^{r} \cup N_{r-1}$ as $P_{d-b-1}^{r} \cup N_{r-1}$ is a proper partial hypergraph of $P_{d-b}^{r}$. Meantime, by Proposition 13, we have

$$
P_{b-1}^{r} \cup P_{d-b}^{r}=P_{\left\lceil\frac{d}{2}\right\rceil-1}^{r} \cup P_{\left\lfloor\frac{d}{2}\right\rfloor}^{r} \succ P_{\left\lceil\frac{d}{2}\right\rceil-2}^{r} \cup P_{\left\lfloor\frac{d}{2}\right\rfloor+1}^{r}=P_{b-2}^{r} \cup P_{d-b+1}^{r} .
$$

Therefore, by Theorems 3, 14 and $(32), \varphi\left(\mathcal{T}^{\prime \prime}, x\right)<\varphi\left(T_{(m, d, r)}(\lceil d / 2\rceil), x\right)$ if $x \geqslant \rho\left(\mathcal{T}^{\prime \prime}\right)$. Consequently, $\mathcal{T}^{\prime \prime} \succ T_{(m, d, r)}(\lceil d / 2\rceil)$.

Lemma 27. For any $\mathcal{T} \in S(m, d, r) \backslash\left\{\hat{T}_{(m, d)}^{r} \cup \mathcal{T}^{\prime \prime}\right\}$ with $m \geqslant d+3$ and $d \geqslant 3$, we have

$$
\rho(\mathcal{T})<\rho\left(\mathcal{T}^{\prime \prime}\right) .
$$

Proof. Choose a supertree $\mathcal{T} \in S(m, d, r) \backslash\left\{\hat{T}_{(m, d)}^{r} \cup \mathcal{T}^{\prime \prime}\right\}$ with the maximum spectral radius. Let $P_{d}^{r}=\left(v_{1}, e_{1}, v_{2}, e_{2}, \ldots, v_{d}, e_{d}, v_{d+1}\right)$ be the diametral path in $\mathcal{T}$. Then $\mathcal{T} \backslash$ $\left\{e_{1}, \ldots, e_{d}\right\}$ is disconnected. Let $\mathcal{T}_{1}, \ldots, \mathcal{T}_{k}$ be the connected components of $\mathcal{T} \backslash\left\{e_{1}, \ldots, e_{d}\right\}$ which are not isolated vertex. Then $\mathcal{T}_{j}$ and $P_{d}^{r}$ share a unique common vertex, say $w_{j}$, for $j=1, \ldots, k$. By Theorem 20 and the maximality of $\mathcal{T}$, we have 
(1) either every $\mathcal{T}_{j}$ is a hyperstar with $w_{j}$ as its center, for $j=1, \ldots, k$.

(2) or there is one of $\mathcal{T}_{1}, \ldots, \mathcal{T}_{k}$, say $\mathcal{T}_{1}$, such that if $\mathcal{T}_{1}$ is a hyperstar with center $w_{1}, \mathcal{T}$ will then become $\mathcal{T}^{\prime \prime}$ or a maximal one in $\hat{T}_{(m, d)}^{r}$.

(1) First consider the former case, that is, every $\mathcal{T}_{j}$ is a hyperstar with $w_{j}$ as its center, for $j=1, \ldots, k$. We distinguish two cases according to $w_{i}(i=1, \ldots, k)$ are contained in $\left\{v_{2}, \ldots, v_{d}\right\}$ or not.

Case 1. $\left\{w_{1}, \ldots, w_{k}\right\} \subseteq\left\{v_{2}, \ldots, v_{d}\right\}$.

Then $\mathcal{T}$ must be an $r$ th power of a tree $T$ of diameter $d$ and size $m$, and $T \in T_{m, d} \backslash$ $\left\{\hat{T}_{(m, d)} \cup T^{\prime \prime}\right\}$. From Lemma 24 and Lemma 5, it follows immediately that

$$
\rho(\mathcal{T})=\rho(T)^{2 / r}<\rho\left(T^{\prime \prime}\right)^{2 / r}=\rho\left(\mathcal{T}^{\prime \prime}\right) .
$$

Case 2. $\left\{w_{1}, \ldots, w_{k}\right\} \nsubseteq\left\{v_{2}, \ldots, v_{d}\right\}$.

If $k=1$, then $w_{1}$ is a vertex of an edge $e_{i}$, and $w_{1} \notin\left\{v_{i}, v_{i+1}\right\}$, where $2 \leqslant i \leqslant d-1$. Then $\mathcal{T} \cong T_{(m, d, r)}(i)$. By Theorem 21 and Lemma 26, we have

$$
\rho(\mathcal{T}) \leqslant \rho\left(T_{(m, d, r)}(\lceil d / 2\rceil)\right)<\rho\left(\mathcal{T}^{\prime \prime}\right) .
$$

If $k \geqslant 2$, without loss of generality, we may assume $w_{1}$ is a vertex of edge $e_{i}$ and $w_{1} \notin\left\{v_{i}, v_{i+1}\right\}$. Denote by $H_{1}$ and $H_{2}$ the supertrees obtained from $\mathcal{T}$ by moving all edges in $E_{w_{1}} \cap E\left(\mathcal{T}_{1}\right)$ from $w_{1}$ to $v_{i}$ and $v_{i+1}$, respectively. By Corollary 23, $\rho(\mathcal{T})<$ $\min \left\{\rho\left(H_{1}\right), \rho\left(H_{2}\right)\right\}$. The maximality of $\rho(\mathcal{T})$ implies that $H_{1}, H_{2} \in\left\{\hat{T}_{(m, d)}^{r} \cup \mathcal{T}^{\prime \prime}\right\}$ and one of them is $\mathcal{T}^{\prime \prime}$. So $\rho(\mathcal{T})<\rho\left(\mathcal{T}^{\prime \prime}\right)$.

(2) In the latter case, clearly either $\mathcal{T}=P_{d}^{r}\left(v_{\lfloor d / 2\rfloor+1}, w_{1}\right) \mathcal{T}_{1}$ or $\mathcal{T}=\tilde{P}_{d}^{r}\left(v_{\lceil d / 2\rceil}, w_{1}\right) \mathcal{T}_{1}$, where $\tilde{P}_{d}^{r}$ denotes the one obtained from $P_{d}^{r}$ by attaching a pendent edge at vertex $v_{\lceil d / 2\rceil+1}$. Assume that $\mathcal{T}_{1}$ has $m_{1}$ edges. By Theorem 20, $\rho\left(\tilde{P}_{d}^{r}\left(v_{\lceil d / 2\rceil}, w_{1}\right) \mathcal{T}_{1}\right)<\rho\left(\tilde{P}_{d}^{r}\left(v_{\lceil d / 2\rceil}, w_{1}\right) S_{m_{1}}^{r}\right)$, where $S_{m_{1}}^{r}$ has $w_{1}$ as its center and $\left.\tilde{P}_{d}^{r}\left(v_{\lceil d / 2\rceil}, w_{1}\right) S_{m_{1}}^{r}\right) \cong \mathcal{T}^{\prime \prime}$.

Now it remains to consider the case that $\mathcal{T}=P_{d}^{r}\left(v_{\lfloor d / 2\rfloor+1}, w_{1}\right) \mathcal{T}_{1}$. Since $\mathcal{T}_{1}$ is not a hyperstar with center $w_{1}$, there exists some edge $e \in E_{w_{1}}\left(\mathcal{T}_{1}\right)$ such that $e$ is a nonpendent edge. Assume that $e$ has intersection vertices $w_{1}, w_{2}, \ldots, w_{l}(l \geqslant 2)$, and $\mathcal{T}_{1}$ (see Fig. 6) has $\mathcal{T}_{i}^{\prime}$ as the attached supertree at $w_{i}$, for $i=1, \ldots, l$. Assume that $\mathcal{T}_{1}^{\prime}$ has $m_{1}^{\prime}$ edges. Let $H_{1}$ (see Fig. 6) denote the supertree obtained by replacing each $\mathcal{T}_{i}^{\prime}$ with a hyperstar of the same size centered at $w_{i}$ for $i=1, \ldots, l$. By Theorem 20, we have $\rho(\mathcal{T}) \leqslant \rho\left(H_{1}\right)$, with equality only if $\mathcal{T}$ has been the form of $H_{1}$. Let $H_{2}$ (see Fig. 6) denote the supertree obtained by moving all pendent edges attached at $w_{2}, \ldots, w_{l}$ to a vertex, say $w_{2}$. By Theorem $4, \rho\left(H_{1}\right) \leqslant \rho\left(H_{2}\right)$, with equality only if $l=2$. Let $\dot{\mathcal{T}}$ and $\ddot{\mathcal{T}}$ (see Fig. 6) denote the supertrees obtained by moving all pendent edges from $w_{1}$ to $w_{2}$ and moving all but one pendent edges from $w_{2}$ to $w_{1}$, respectively. By Theorem 4 , we have $\rho\left(H_{2}\right) \leqslant \max \{\rho(\dot{\mathcal{T}}), \rho(\ddot{\mathcal{T}})\}$. By the maximality of $\mathcal{T}$ in $S(m, d, r) \backslash\left\{\dot{T}_{(m, d)}^{r} \cup \mathcal{T}^{\prime \prime}\right\}, \mathcal{T}$ should be $\dot{\mathcal{T}}$ or $\ddot{\mathcal{T}}$. However, both $\dot{\mathcal{T}}$ and $\ddot{\mathcal{T}}$ are $r$ th power of respective trees of diameter $d$ and size $m$. Similarly as in Case 1 , we have $\max \{\rho(\dot{\mathcal{T}}), \rho(\ddot{\mathcal{T}})\}<\rho\left(\mathcal{T}^{\prime \prime}\right)$. The proof is finished. 


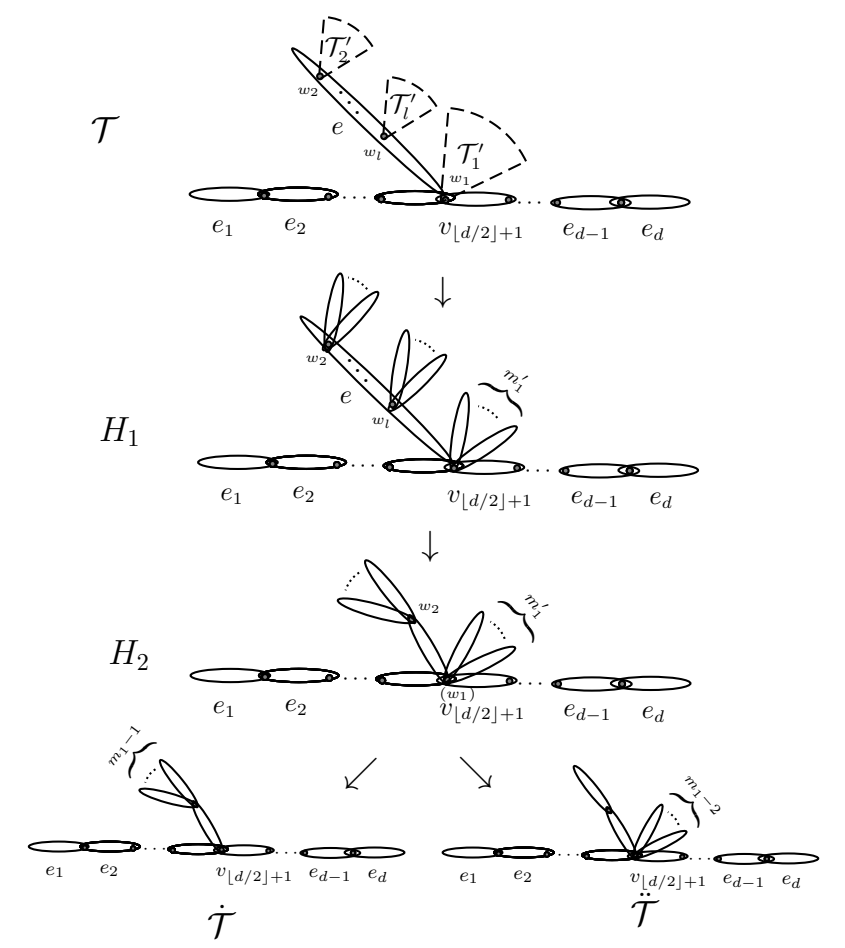

Figure 6: Supertrees $\mathcal{T}, H_{1}, H_{2}, \dot{\mathcal{T}}$ and $\ddot{\mathcal{T}}$.

By Theorems 5, 25 and Lemma 27, we have the following results.

Theorem 28. The first $\left\lfloor\frac{d}{2}\right\rfloor+1$ largest spectral radii of supertrees in the set $S(m, d, r)$ with $m \geqslant d+3$ and $d \geqslant 3$ are $T_{(m, d)}^{r}(\lfloor d / 2\rfloor+1), T_{(m, d)}^{r}(\lfloor d / 2\rfloor), \ldots, T_{(m, d)}^{r}(3), T_{(m, d)}^{r}(2), \mathcal{T}^{\prime \prime}$.

Theorem 29. The first $\left\lfloor\frac{d}{2}\right\rfloor-1$ largest spectral radii of supertrees in the set $S(m, d, r)$ with $m=d+2$ and $d \geqslant 4$ are $T_{(m, d)}^{r}(\lfloor d / 2\rfloor+1), T_{(m, d)}^{r}(\lfloor d / 2\rfloor), \ldots, T_{(m, d)}^{r}(3)$.

\section{The second minimal supertree}

Let $P_{m-1}^{r}=\left(v_{1}, e_{1}, v_{2}, e_{2}, \ldots, e_{m-1}, v_{m}\right)$ be a loose path of length $m-1$. Denote by $D_{m, r}$ the supertree obtained from $P_{m-1}^{r}$ by attaching a pendent edge at a core vertex of $e_{2}$ (see Fig. 7(a)). Let $\grave{P}_{m}^{r}$ be the supertree obtained from $P_{m-1}^{r}$ by attaching a pendent edge at the vertex $v_{2}$ (see Fig. $7(\mathrm{~b})$ ). We use $S(m, r)$ to denote the set of $r$-uniform supertrees with $m$ edges.

Theorem 30. Any $r$-uniform supertree $\mathcal{T}$ with $m(m \geqslant 4)$ edges different from $P_{m}^{r}$ satisfies $\mathcal{T} \succeq D_{m, r}$.

Proof. Choose a supertree $\mathcal{T}_{0}$ from $S(m, r) \backslash\left\{P_{m}^{r}\right\}$ such that $\mathcal{T}_{0} \preceq \mathcal{T}$ for any $\mathcal{T} \in S(m, r) \backslash$ $\left\{P_{m}^{r}\right\}$. Then $\mathcal{T}_{0}$ either has a vertex of degree more than two or has an edge with at least three intersection vertices. We consider the two cases as follows. 
(a)

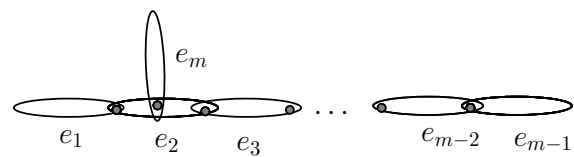

(b)

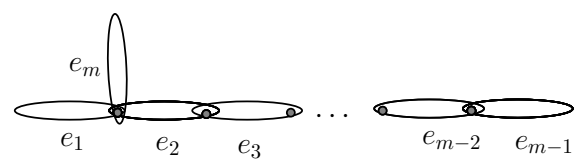

Figure 7: Supertrees $(a) D_{m, r} ; \quad(b) \grave{P}_{m}^{r}$.

Case 1. There exists a vertex of degree greater than two, say $v \in V\left(\mathcal{T}_{0}\right)$ with $\operatorname{deg}(v) \geqslant 3$. Thus $\mathcal{T}_{0}$ can be described as a supertree in the form of some supertrees, say $\mathcal{T}_{1}, \ldots, \mathcal{T}_{s}$ $(s \geqslant 3)$, attached at a single vertex $v$. Denote $\mathcal{T}_{0}$ by $\mathcal{T}_{1}(v) \mathcal{T}_{2}(v) \cdots(v) \mathcal{T}_{s}$ (see Fig. 8(a)). Assume that $\mathcal{T}_{i}$ has $m_{i}$ edges for $i=1, \ldots, s$. Let $m^{\prime}=m-\left(m_{1}+m_{2}\right)$. By Theorems 20 and 15 , we have

$$
\mathcal{T}_{1}(v) \mathcal{T}_{2}(v) \cdots(v) \mathcal{T}_{s} \succeq P_{m_{1}}^{r}(v) P_{m_{2}}^{r}(v) \cdots(v) P_{m_{s}}^{r} \succeq P_{m_{1}}^{r}(v) P_{m_{2}}^{t}(v) P_{m^{\prime}}^{r} \succeq P_{1}^{r}(v) P_{1}^{r}(v) P_{m-2}^{r},
$$

where all loose paths $P_{m^{\prime}}^{r}, P_{m-2}^{r}$ and $P_{m_{j}}^{r}(j=1, \ldots, s)$ have $v$ as its end vertex. By the minimality of $\mathcal{T}_{0}, \mathcal{T}_{0}=P_{1}^{r}(v) P_{1}^{r}(v) P_{m-2}^{r}=\grave{P}_{m}^{r}$.

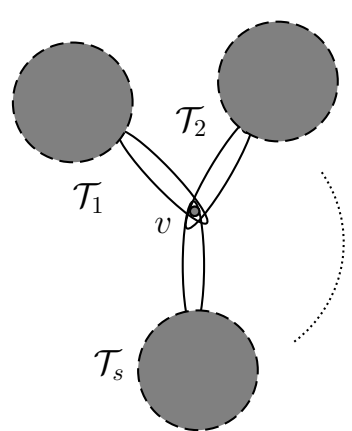

(a)

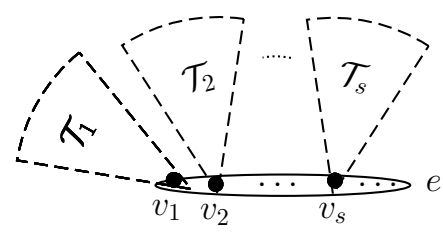

(b)

Figure 8: Supertrees $(a)$ and $(b)$.

Case 2. There exists an edge $e$ of $\mathcal{T}_{0}$ with at least three intersection vertices. Without loss of generality, assume that $e=\left\{v_{1}, \ldots, v_{r}\right\}$ and $\operatorname{deg}\left(v_{i}\right) \geqslant 2$ for $i=1, \ldots, s(3 \leqslant s \leqslant r)$, and other vertices in $e$ (if any) are core vertices (see Fig. 8(b)). Then $\mathcal{T}_{0}$ may be viewed as having been obtained by attaching supertrees, say $\mathcal{T}_{1}, \ldots, \mathcal{T}_{s}$, at $v_{1}, \ldots, v_{s}$ respectively.

By Theorems 16, 20 and the minimality of $\mathcal{T}_{0}$, the following conclusions hold.

(1) $\mathcal{T}_{1}, \ldots, \mathcal{T}_{s}$ are pendent paths attached at $v_{1}, \ldots, v_{s}$ respectively.

(2) $s=3$.

(3) Two of $\mathcal{T}_{1}, \mathcal{T}_{2}, \mathcal{T}_{3}$ are of length one. 
Therefore, $\mathcal{T}_{0}=D_{m, r}$.

Combining two cases above, we have shown that $\mathcal{T}_{0} \in\left\{\grave{P}_{m}^{r}, D_{m, r}\right\}$. Further by (a) of Corollary 23, we have $\grave{P}_{m}^{r} \succ D_{m, r}$. So $\mathcal{T}_{0}=D_{m, r}$. Thus we conclude that for any $\mathcal{T} \in S(m, r) \backslash\left\{P_{m}^{r}\right\}, \mathcal{T} \succeq D_{m, r}$.

Theorem 31. The first two smallest spectral radii of supertrees with $m(m \geqslant 4)$ edges are $P_{m}^{r}, D_{m, r}$.

\section{Closing remarks}

We conclude this section with some remarks on matching polynomial of a supertree. The work in this paper is based on the relation between the roots of matching polynomial of a supertree and its spectrum developed in [34]. Using the recurrence relations of matching polynomial of supertrees, the effect on the spectral perturbation of supertree by grafting edges in various situations can be explained. The methods are initially used to compare spectral radii of supertrees in this paper. The methods are shown to be efficient in dealing with extremal supertrees with respect to their spectral radii, such as in finding the first two smallest supertrees and the first several largest supertrees with given diameter.

For the corresponding problem on a hypergraph, the characteristic polynomial of adjacency tensor of a hypergraph might be used to compare spectral radii of hypergraphs. However, the degree of characteristic polynomial of a hypergraph is very high relative to its order, and very little is known about it up to now. Finally, we pose the following problem.

Problem 32. What kind of polynomial should be associated with a hypergraph satisfying the following conditions:

(1) The roots of the associated polynomial consist of the eigenvalues, especially the spectral radius of the hypergraph.

(2) The coefficients of the polynomial reflect certain structural information of the hypergraph, such as matching, cyclic structure or something more complicated.

\section{References}

[1] A. Bretto. Hypergraph Theory: An Introduction. Springer, 2013.

[2] R. Brualdi, E. Solheid. On the spectral radius of complementary acyclic matrices of zeros and ones. SIAM J. Algebraic Discrete Methods, 7:265-272, 1986.

[3] K. Chang, K. Pearson, T. Zhang. Perron-Frobenius theorem for nonnegative tensors. Commun. Math. Sci., 6:507-520, 2008.

[4] K. Chang, L. Qi, T. Zhang. A survey on the spectral theory of nonnegative tensors. Numer. Linear Alg. Appl., 20:891-912, 2013.

[5] D. Chen, Z. Chen, X. Zhang. Spectral radius of uniform hypergraphs and degree sequences. Front. Math. China, 12:1279-1288, 2017. 
[6] G. Clark, J. Cooper. On the adjacency spectra of hypertrees. Electron. J. Combin., 25:\#P2.48, 2018.

[7] L. Collatz, U. Sinogowitz. Spektren endlicher Grafen. Abh. Math. Sem. Univ. Hamburg, 21:63-77, 1957.

[8] J. Cooper, A. Dutle. Spectra of uniform hypergraphs. Linear Algebra Appl., 436:3268-3292, 2012.

[9] S. Friedland, A. Gaubert, L. Han. Perron-Frobenius theorems for nonnegative multilinear forms and extensions. Linear Algebra Appl., 438:738-749, 2013.

[10] J. Guo, J. Shao. On the spectral radius of trees with fixed diameter. Linear Algebra Appl., 413:131-147, 2006.

[11] Y. Hou, A. Chang, L. Zhang. Largest $H$-eigenvalue of uniform $s$-hypertrees. Front. Math. China, 13:301-312, 2018.

[12] S. Hu, L. Qi. The Laplacian of a uniform hypergraph. J. Comb. Optim., 29:331-366, 2015.

[13] S. Hu, L. Qi, J. Shao. Cored hypergraphs, power hypergraphs and their Laplacian eigenvalues. Linear Algebra Appl., 439:2980-2998, 2013.

[14] L. Kang, V. Nikiforov, X. Yuan. The $p$-spectral radius of $k$-partite and $k$-chromatic uniform hypergraphs. Linear Algebra Appl., 478:81-107, 2015.

[15] M. Khan, Y. Fan. On the spectral radius of a class of non-odd-bipartite even uniform hypergraphs. Linear Algebra Appl., 480:93-106, 2015.

[16] H. Li, J. Shao, L. Qi. The extremal spectral radii of $k$-uniform supertrees. J. Comb. Optim., 32:741-764, 2016.

[17] Q. Li, K. Feng. On the largest eigenvalue of a graph. Acta Math. Appl. Sinica, 2:167-175, 1979.

[18] L. Lim. Singular values and eigenvalues of tensors: a variational approach. In: Proceedings of the IEEE International Workshop on Computational Advances in MultiSensor Adaptive Processing (CAMSAP'05), vol. 1:129-132, 2005.

[19] L. Lim. Eigenvalues of tensors and some very basic spectral hypergraph theory. Matrix compu-tations and scientific computing seminar. http://www. stat.uchicago. edu/lekheng/work/mcsc2.pdf, April 16, 2008.

[20] H. Lin, B. Zhou, B. Mo. Upper bounds for $H$ - and $Z$-spectral radii of uniform hypergraphs. Linear Algebra Appl., 510:205-221, 2016.

[21] L. Lovász, J. Pelikán. On the eigenvalues of trees. Period. Math. Hungar., 3:175-182, 1973.

[22] L. Lu, S. Man. Connected hypergraphs with small spectral radius. Linear Algebra Appl., 509:206-227, 2016.

[23] V. Nikiforov. Analytic methods for uniform hypergraphs. Linear Algebra Appl., 457:455-535, 2014. 
[24] L. Qi. Eigenvalues of a real supersymmetric tensor. J. Symb. Comput., 40:1302-1324, 2005.

[25] L. Qi, Z. Luo. Tensor Analysis: Spectral Theory and Special Tensors. Siam, 2017.

[26] J. Y. Shao. A general product of tensors with applications. Linear Algebra Appl., 439:2350-2366, 2013.

[27] P. Xiao, L. Wang, Y. Lu. The maximum spectral radii of uniform supertrees with given degree sequences. Linear Algebra Appl., 523:33-45, 2017.

[28] P. Xiao, L. Wang, Y. Du. The first two largest spectral radii of uniform supertrees with given diameter. Linear Algebra Appl., 536:103-119, 2018.

[29] P. Xiao, L. Wang. The maximum spectral radius of uniform hypergraphs with given number of pendant edges. Linear and Multilinear Algebra., https://doi.org/10. 1080/03081087.2018.1453471, 2018.

[30] Q. Yang, Y. Yang. Further results for Perron-Frobenius theorem for nonnegative tensors II. SIAM J. Matrix Anal. Appl., 32:1236-1250, 2011.

[31] Y. Yang, Q. Yang. Further results for Perron-Frobenius theorem for nonnegative tensors. SIAM J. Matrix Anal. Appl., 31:2517-2530, 2010.

[32] Y. Yang, Q. Yang. On some properties of nonnegativeweakly irreducible tensors. arXiv:1111.0713v3, 2011.

[33] X. Yuan, J. Shao, H. Shan. Ordering of some uniform supertrees with larger spectral radii. Linear Algebra Appl., 495:206-222, 2016.

[34] W. Zhang, L. Kang, E. Shan, Y. Bai. The spectra of uniform hypertrees. Linear Algebra Appl., 533:84-94, 2017.

[35] J. Zhou, L. Sun, W. Wang, C. Bu. Some spectral properties of uniform hyper-graphs. Electron. J. Combin., 21: \#P4.24, 2014. 